\title{
Studies in the Basidiodendron caesiocinereum complex (Auriculariales, Basidiomycota)
}

\author{
Viacheslav Spirin ${ }^{1}$ (D) Vera Malysheva ${ }^{2} \cdot$ Nathan Schoutteten $^{3} \cdot$ Ilya Viner $^{1} \cdot$ Otto Miettinen $^{1} \cdot$ Jenni Nordén ${ }^{4}$.

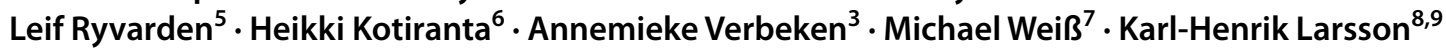

Received: 28 April 2021 / Revised: 22 June 2021 / Accepted: 23 June 2021

(C) The Author(s) 2021

\begin{abstract}
Taxonomy of Basidiodendron caesiocinereum complex is revised based on morphological and molecular methods (with the use of nc LSU rDNA, ITS and TEF1 regions). The basidiospore ornamentation is justified as a key morphological character for the species recognition in the group. As redefined here, B. caesiocinereum is an angiosperm-dwelling species with smooth basidiospores. Bourdotia cinerella and B. cinerella var. trachyspora are proved to represent separate species with warted basidiospores; they are reintroduced as Basidiodendron cinerellum and B. trachysporum. Additionally, eight new species related to B. caesiocinereum are described based on material from Eurasia, North America and Africa, and identity of $B$. spinosum from Oceania is discussed.
\end{abstract}

Keywords Basidiospores $\cdot$ Heterobasidiomycetes $\cdot$ Phylogeny $\cdot$ Taxonomy

\section{Introduction}

Section Editor: Yu-Cheng Dai

Viacheslav Spirin

viacheslav.spirin@helsinki.fi

1 Finnish Museum of Natural History, University of Helsinki, PO Box 7, FI-00014 Helsinki, Finland

2 Komarov Botanical Institute RAS, 2 Prof. Popov str., St. Petersburg 197376, Russia

3 Department of Biology, Ghent University, K.L. Ledeganckstraat 35, B-9000 Gent, Belgium

4 Norwegian Institute for Nature Research, Gaustadalléen 21, 0349 Oslo, Norway

5 Department of Biosciences, University of Oslo, Blindern, P.O. Box 1066, 0316 Oslo, Norway

6 Finnish Environment Institute, Latokartanonkaari 11, 00790 Helsinki, Finland

7 Steinbeis-Innovationszentrum, Organismische Mykologie und Mikrobiologie, Vor dem Kreuzberg 17, 72070 Tübingen, Germany

8 Natural History Museum, University of Oslo, Blindern, P.O. Box 1172, 0318 Oslo, Norway

9 Gothenburg Global Biodiversity Centre, Post Box 461, 40530 Gothenburg, Sweden
For almost two centuries, the basidiospore features, e.g. their shape, size, colour and ornamentation, have remained among the main features for morphological recognition of the basidiomycetous taxa. Almost all members of the Auriculariales (Basidiomycota) have small or medium-sized, colourless (hyaline), smooth basidiospores and three species with ornamented (warted or spiny) basidiospores have been so far detected in the genus Basidiodendron Rick (Wojewoda 1981). Two of them, Basidiodendron asperum (L.S. Olive) Wojewoda and B. spinosum (L.S. Olive) Wojewoda, are known from their type localities in Tahiti, while the third one, B. caesiocinereum (Höhn. \& Litsch.) Luck-Allen, was reported from different geographic areas of the world (Wells and Raitviir 1975).

As re-defined by Luck-Allen (1963), the genus Basidiodendron Rick embraces effused wood-inhabiting fungi with smooth or minutely warted hymenophore, prominent gloeocystidia and longitudinally septate, predominantly fourcelled basidia. It differs from the morphologically similar genus Bourdotia (Bres.) Bres. \& Torrend in having waxy or arid, non-hygroscopic basidiocarps and on average shorter basidia devoid of a basal stalk (Luck-Allen 1963, Wells and Raitviir 1975). Recent phylogenetic studies confirmed that Basidiodendron and Bourdotia should be treated as separate 
genera (Weiß and Oberwinkler 2001, Spirin et al. 2020). While re-describing B. caesiocinereum, Wells (1959) and Luck-Allen (1963) noted it may have smooth or ornamented basidiospores. McNabb (1969) and Gilbertson (1974) found this feature taxonomically insignificant. On the contrary, Oberwinkler (1963) designated warted-spored collections as Bourdotia caesiocinerea (Höhn. \& Litsch.) Bourdot \& Galzin var. trachyspora and clearly separated them from the smooth-spored $B$. caesiocinerea s. str. Based on nrLSU sequences, Weiß and Oberwinkler (2001) showed these taxa are not conspecific and they belong to Basidiodendron, although no explicit taxonomic conclusions were proposed. Finally, Roberts (2001) subsumed the warted-spored specimens of B. caesiocinereum under B. spinosum. To investigate this problem more closely, we examined type specimens of Corticium caesiocinereum Höhn. \& Litsch. (= Basidiodendron caesiocinereum) and Sebacina spinosa L.S. Olive $(=B$. spinosum), as well as authentic material of Bourdotia cinerella Bourdot \& Galzin (incl. var. trachyspora) (to date, a synonym of $B$. caesiocinereum) and a number of B. caesiocinereum $\mathrm{s}$. lato specimens from boreal - temperate Eurasia, North America (USA and Mexico) and Africa (Malawi). Additionally, a DNA study based on three markers (nc LSU rDNA, ITS and TEF1) was conducted. Results of this study are presented below.

\section{Material and methods}

\section{Morphological study}

Type specimens and collections from herbaria $\mathrm{H}, \mathrm{O}$, GENT, LE, FH, PC, NY, TAAM, TU, CWU were studied. Herbarium acronyms are given according to Thiers (2020). Microscopic routine and terminology follow Spirin et al. (2020). All measurements were made from microscopic slides mounted in Cotton Blue, using phase contrast and oil immersion lens (Leitz Diaplan microscope, $\times 1250$ magnification). At least 20 basidia, 10-20 gloeocystidia and 20-30 basidiospores were measured for each specimen studied. The following abbreviations are used in the taxonomic section: $\mathrm{L}$, mean basidiospore length; W, mean basidiospore width; Q', $\mathrm{L} / \mathrm{W}$ ratio; $\mathrm{Q}$, mean $\mathrm{L} / \mathrm{W}$ ratio; and $\mathrm{n}$, number of measurements per specimens measured. Advances of phase contrast illumination versus bright-field microscopy are explained in Stein (1969). For microscopic study, we subjectively determined an accuracy of measurements as $0.1 \mu \mathrm{m}$. These measurements (including those for the basidiospore ornamentation elements, i.e. warts or spines) were subsequently calibrated with the scanning electronic microscope (SEM). For preparing SEM photos, basidiocarps were coated with a 25-mm layer of gold-palladium using an Eiko IB-3 sputter coater. Micrographs were taken using JSM-6380LA microscope at the Moscow State University (Russia) and JEOL JSM-7100FLV field emission microscope at Botanical Garden Meise (Belgium).

\section{DNA study}

In total, 80 specimens were selected for molecular sampling (Table 1). We performed PCR directly from small fragments of dried basidiocarps (without prior DNA extraction) using Phire Plant Direct PCR Kit (Thermo Scientific) according to the manufacturer's instructions. DNA extraction of Belgian and Dutch collections was done using the DNeasy Plant Mini kit (Qiagen).

The following primers were used for both amplification and sequencing: the primers ITS1F (Gardes and Bruns 1993) and ITS4 (White et al. 1990) for the nrITS1-5.8SITS2 region, primers EF1-983F and EF1-1567R (Rehner and Buckley 2005) for a part of the TEF1 region and primers JS1 (Landvik 1996) and LR5 (Vilgalys and Hester 1990) for D1-D3 domains of nc LSU rDNA region. PCR products were purified applying the GeneJET Gel Extraction and DNA Cleanup Micro Kit (Thermo Scientific). Sequencing was performed with an ABI model 3500 Genetic Analyzer (Applied Biosystems). Raw data were edited and assembled in MEGA 7 (Kumar et al. 2018). Molecular studies were mostly carried out at the centre for collective use of scientific equipment "Cellular and molecular technology of studying plants and fungi" (Komarov Botanical Institute, Russian Academy of Sciences, St. Petersburg, Russia) and the centre for molecular phylogeny and evolution $(\mathrm{CeMoFe})$ (Ghent University, Belgium).

For this study, 76 ITS, 30 TEF1 and $35 \mathrm{nc}$ LSU rDNA sequences were generated (Table 1). Additionally, 71 ITS and $61 \mathrm{nc}$ LSU rDNA sequences, including the outgroup, were retrieved from GenBank and UNITE (www.ncbi. nlm.nih.gov/genbank/; https://unite.ut.ee/). Sequences were aligned with the MAFFT version 7 web tool (http:// mafft.cbrc.jp/alignment/server/) with subsequent manual processing.

Three datasets were prepared for the present study: (1) ITS + nc LSU rDNA dataset (1647 characters including gaps) used to reconstruct a general topology of the Auriculariales, with special focus on Basidiodendron spp.; (2) ITS + nc LSU rDNA + TEF1 (1884 characters including gaps); and (3) ITS only (628 characters including gaps) datasets for the $B$. caesiocinereum complex.

Phylogenetic reconstructions were performed with maximum likelihood (ML) and Bayesian (BI) analyses. Before the analyses, the best-fit substitution model for the alignment was estimated based on the Akaike Information Criterion (AIC) using FindModel web server (http://www.hiv.lanl. gov/content/sequence/findmodel/findmodel.html). "K80 plus Gamma" model was chosen for ITS dataset, and "GTR 
Table 1 DNA sequences generated for the present study

\begin{tabular}{|c|c|c|c|c|c|c|}
\hline \multirow[t]{2}{*}{ Species } & \multirow[t]{2}{*}{ Specimen/herbarium } & \multirow[t]{2}{*}{ Country (ISO code) } & \multirow[t]{2}{*}{ Host } & \multicolumn{3}{|c|}{ GenBank / UNITE sequence numbers } \\
\hline & & & & nrITS & nrLSU & TEF1 \\
\hline $\begin{array}{l}\text { Basidiodendron } \\
\text { caesiocinereum }\end{array}$ & NS 18-172 (GENT) & $\mathrm{BE}$ & Fagus sylvatica & MW139272 & - & - \\
\hline B. caesiocinereum & NS 18-226 (GENT) & $\mathrm{BE}$ & F. sylvatica & MW139267 & - & - \\
\hline B. caesiocinereum & NS 18-625 (GENT) & $\mathrm{BE}$ & F. sylvatica & MW139268 & - & - \\
\hline B. caesiocinereum & NS 18-902 (GENT) & $\mathrm{BE}$ & F. sylvatica & MW139269 & - & - \\
\hline B. caesiocinereum & NS 18-925 (GENT) & $\mathrm{BE}$ & F. sylvatica & MW139270 & - & - \\
\hline B. caesiocinereum & NS 18-1050 (GENT) & $\mathrm{BE}$ & F. sylvatica & MW139266 & - & - \\
\hline B. caesiocinereum & NS 18-1051 (GENT) & $\mathrm{BE}$ & F. sylvatica & MW139271 & - & - \\
\hline B. caesiocinereum & OM $10608(\mathrm{H})$ & $\mathrm{CN}$ & Populus sp. (?) & MW136071 & - & - \\
\hline B. caesiocinereum & VS $13663(\mathrm{H})$ & IT & Corylus avellana & MW136105 & MW136140 & MW187110 \\
\hline B. caesiocinereum & VS $11764(\mathrm{O})$ & NO & Ulmus glabra & MW136082 & - & - \\
\hline B. caesiocinereum & VS $11776(\mathrm{O})$ & NO & Betula pubescens & MW136101 & MW136136 & MW187106 \\
\hline B. caesiocinereum & VS $12536(\mathrm{O})$ & NO & Tilia cordata & MW136069 & - & MW187084 \\
\hline B. caesiocinereum & VS $12500(\mathrm{O})$ & NO & C. avellana & MW136061 & - & - \\
\hline B. caesiocinereum & VS $12502(\mathrm{O})$ & NO & U. glabra & MW136084 & - & MW187095 \\
\hline B. caesiocinereum & VS $12504(\mathrm{O})$ & NO & U. glabra & MW136065 & - & MW187082 \\
\hline B. caesiocinereum & VS $12511(\mathrm{O})$ & NO & U. glabra & MW136083 & - & MW187094 \\
\hline B. caesiocinereum & VS $12515(\mathrm{O})$ & NO & U. glabra & MW136100 & MW136135 & MW187105 \\
\hline B. caesiocinereum & VS $12465(\mathrm{O})$ & NO & U. glabra & MW136102 & MW136137 & MW187107 \\
\hline B. caesiocinereum & VS $11115(\mathrm{O})$ & NO & Alnus incana & MW136103 & MW136138 & MW187108 \\
\hline B. caesiocinereum & SS $901(\mathrm{O})$ & NO & Picea abies & MW259231 & - & - \\
\hline B. caesiocinereum & HK 26428 (H) & RU-KRA & Alnus hirsuta & MW136072 & - & MW187085 \\
\hline B. cinerellum & VS $13485(\mathrm{H})$ & $\mathrm{BE}$ & Pinus sylvestris & MW136088 & MW136125 & MW187097 \\
\hline B. cinerellum & VS 12337 (TU) & $\mathrm{EE}$ & P. sylvestris & UDB0754270 & - & - \\
\hline B. cinerellum & VS 12350 (TU) & $\mathrm{EE}$ & C. avellana & UDB0754279 & - & - \\
\hline B. cinerellum & VS $13681(\mathrm{H})$ & IT & P. abies & MW136086 & MW136123 & MW187096 \\
\hline B. cinerellum & VS $11188(\mathrm{O})$ & NO & A. incana & MW136104 & MW136139 & MW187109 \\
\hline B. cinerellum & VS $12449(\mathrm{O})$ & NO & U. glabra & MW136064 & - & MW187081 \\
\hline B. cinerellum & VS $13317(\mathrm{H})$ & SI & Pinus mиgo & MW136097 & MW136131 & MW187102 \\
\hline B. cinerellum & VS $13275(\mathrm{H})$ & SI & P. тияо & MW136093 & MW136130 & MW187101 \\
\hline B. eyrei & ENZ 13-100 (GENT) & NL & P. abies & MW139273 & - & - \\
\hline B. eyrei & ENZ 18-101 (GENT) & NL & decayed wood & MW139274 & - & - \\
\hline B. eyrei & ENZ 18-103 (GENT) & NL & decayed wood & MW139275 & - & - \\
\hline B. eyrei & NS 19-411 (GENT) & NL & decayed wood & MW139277 & - & - \\
\hline B. glaucum & VS $11750(\mathrm{O})$ & NO & A. incana & MW136085 & - & - \\
\hline B. glaucum & JN 9815 (O) & NO & Picea abies & MW259227 & - & - \\
\hline B. glaucum & JN 9920 (O) & NO & P. abies & MW259234 & - & - \\
\hline B. glaucum & JN 9858 (O) & NO & P. abies & MW259226 & - & - \\
\hline B. glaucum & SS $370(\mathrm{O})$ & NO & P. abies & MW136079 & MW136120 & MW187091 \\
\hline B. glaucum & JN 9683 (O) & NO & P. abies & MW259233 & - & - \\
\hline B. glaucum & JN 9079 (O) & NO & P. abies & MW259232 & - & - \\
\hline B. glaucum & JN 9080 (O) & NO & P. abies & MW259228 & - & - \\
\hline B. glaucum & SS $11(\mathrm{O})$ & NO & P. abies & MW259229 & - & - \\
\hline B. glaucum & SS $140(\mathrm{O})$ & NO & P. abies & MW259235 & - & - \\
\hline B. glaucum & SS $144(\mathrm{O})$ & NO & P. abies & MW136070 & - & - \\
\hline B. glaucum & SS $863(\mathrm{O})$ & NO & P. abies & MW136078 & MW136119 & MW187090 \\
\hline B. glaucum & VS $7890(\mathrm{H})$ & RU-KHA & Picea ajanensis & MW136063 & - & - \\
\hline B. groningae & GVA 20-040 (GENT) & $\mathrm{BE}$ & rotten wood & MW139280 & - & - \\
\hline
\end{tabular}


Table 1 (continued)

\begin{tabular}{|c|c|c|c|c|c|c|}
\hline \multirow[t]{2}{*}{ Species } & \multirow[t]{2}{*}{ Specimen/herbarium } & \multirow[t]{2}{*}{ Country (ISO code) } & \multirow[t]{2}{*}{ Host } & \multicolumn{3}{|c|}{ GenBank / UNITE sequence numbers } \\
\hline & & & & nrITS & nrLSU & TEF1 \\
\hline B. groningae & ENZ 18-001 (GENT) & NL & conifer & MW139278 & MW136483 & - \\
\hline B. groningae & ENZ 19-073 (GENT) & NL & conifer & MW139276 & MW136482 & - \\
\hline B. groningae & NS 18-1325 (GENT) & NL & Hippophae rhamnoides & MW139265 & - & - \\
\hline B. inconspicuum & VS $8171(\mathrm{H})$ & US-WA & Thuja plicata & MW136098 & MW136132 & MW187103 \\
\hline B. mexicanum & LR $23131(\mathrm{O})$ & MX & Pinus patula & MW136068 & - & - \\
\hline B. robenae & OM $16910.2(\mathrm{H})$ & US-NY & hardwood & MW270998 & MW271001 & - \\
\hline B. robenae & OM $19650(\mathrm{H})$ & US-NY & hardwood & MW270997 & MW271000 & - \\
\hline B. spiculosum & LR $23324(\mathrm{O})$ & MX & Cyathea sp. & MW136076 & MW136117 & MW187088 \\
\hline B. trachysporum & TU 112986 & $\mathrm{EE}$ & decayed wood & UDB016299 & - & - \\
\hline B. trachysporum & OM $22962.2(\mathrm{H})$ & FI & P. abies & MW136096 & - & - \\
\hline B. trachysporum & ENZ 20-005 (GENT) & NL & P. sylvestris & MW139281 & - & - \\
\hline B. trachysporum & VS $11111(\mathrm{O})$ & NO & P. abies & MW136089 & MW136126 & - \\
\hline B. trachysporum & VS $12528(\mathrm{O})$ & NO & P. abies & MW136067 & MW136113 & MW187083 \\
\hline B. trachysporum & VS $11801(\mathrm{O})$ & NO & P. abies & MW136091 & MW136129 & MW187100 \\
\hline B. trachysporum & VS $11803(\mathrm{O})$ & NO & P. abies & MW136090 & MW136127 & MW187098 \\
\hline B. trachysporum & VS $12508(\mathrm{O})$ & NO & P. abies & MW136081 & MW136122 & MW187093 \\
\hline B. trachysporum & JN $9601(\mathrm{O})$ & NO & P. abies & MW259230 & MW136134 & - \\
\hline B. trachysporum & SS $608(O)$ & NO & P. abies & MW136062 & MW136111 & - \\
\hline B. trachysporum & HK $26387(\mathrm{H})$ & RU-KRA & Pinus sibirica & MW136087 & MW136124 & - \\
\hline B. trachysporum & VS $11886(\mathrm{H})$ & RU-LEN & P. abies & MW152419 & MW136128 & MW187099 \\
\hline B. trachysporum & VS 9188a (H) & RU-NIZ & P. sylvestris & MW136080 & MW136121 & MW187092 \\
\hline B. trachysporum & VS $9481(\mathrm{H})$ & RU-NIZ & P. sylvestris & MW136074 & MW136115 & MW187086 \\
\hline B. trachysporum & VS $9483(\mathrm{H})$ & RU-NIZ & U. glabra & MW136077 & MW136118 & MW187089 \\
\hline B. trachysporum & HK $29616(H)$ & RU-ZAB & conifer & MW270996 & MW270999 & - \\
\hline B. trachysporum & VS $12548(\mathrm{H})$ & SI & Larix decidua & MW136099 & MW136133 & MW187104 \\
\hline B. trachysporum & VS $12623(\mathrm{H})$ & SI & P. abies & MW136095 & - & - \\
\hline B. trachysporum & VS $13147(\mathrm{H})$ & SI & P. abies & MW136092 & - & - \\
\hline B. trachysporum & AS 72 (CWU) & UA & Quercus robur & MW136094 & - & - \\
\hline B. trachysporum & VS $8262(\mathrm{H})$ & US-WA & Pseudotsuga menziesii & MW136060 & - & - \\
\hline B. trachysporum & VS $8740(\mathrm{H})$ & US-WA & Abies lasiocarpa & MW136075 & MW136116 & MW187087 \\
\hline B. walleynii & WR 3081 (GENT) & $\mathrm{BE}$ & Q. robur & MW139279 & - & - \\
\hline B. walleynii & VS $9697(\mathrm{H})$ & RU-NIZ & Q. robur & MW136066 & MW136112 & - \\
\hline B. widdringtoniae & LR 11307a (O) & MW & Widdringtonia whytei & MW136073 & MW136114 & - \\
\hline
\end{tabular}

plus Gamma" model was chosen for nrITS + nc LSU rDNA + TEF 1 and ITS + nc LSU rDNA datasets.

Maximum likelihood analysis was run on RAxML servers, v.0.9.0 (Kozlov et al. 2019) with 1000 rapid bootstrap replicates. Bayesian analyses was performed with MrBayes 3.2.5 software (Ronquist et al. 2012), for two independent runs, each with 5 million generations (for ITS + nc LSU rDNA + TEF 1 dataset) and 10 million generations (for ITS and ITS + nc LSU rDNA datasets), under described models and four chains with sampling every 100 generations. To check for convergence of MCMC analyses and to get estimates of the posterior distribution of parameter values Tracer v1.6 was used (Rambaut et al. 2014). We accepted the result where the ESS (effective sample size) was above 200 and the PSRF (potential scale reduction factor) was close to 1 .

The outgroup choice for the order-level phylogeny (Sistotrema brinkmannii (Bres.) J. Erikss., Cantharellales) was guided by the current JGI Basidiomycota tree ((https:// mycocosm.jgi.doe.gov/mycocosm/species-tree/tree; FJDxL?organism=basidiomycota) where Cantharellales were recovered close to Auriculariales and Sebacinales. ITS, nc LSU rDNA and TEF1 sequences of Bourdotia, the sister genus of Basidiodendron, were selected as outgroups for the species-level analyses of the $B$. caesiocinereum complex.

Newly generated sequences have been deposited in GenBank with corresponding accession numbers (Table 1). Alignments have been deposited in TreeBASE (S27231). 


\section{Results}

\section{ITS + nc LSU rDNA dataset}

The overall topologies of the ML and BI trees were nearly identical (Fig. 1). They uncovered all B. caesiocinereum s. lato specimens involved in the analyses in one strongly supported clade ( $\mathrm{bs}=100, \mathrm{pp}=1)$ with $B$. luteogriseum Rick (the generic type of Basidiodendron) and members of the B. eyrei complex (as defined by Spirin et al. 2020). Therefore, we interpreted all these taxa as belonging to one genus, Basidiodendron. In turn, the Basidiodendron clade was split into four strongly supported subclades. Two of them covered the Basidiodendron eyrei complex and contained fourteen species with smooth, ellipsoid or globose, usually small basidiospores. They all were dealt with in our previous publication (Spirin et al. 2020). Two remaining subclades encompassed the $B$. caesiocinereum complex. The larger subclade (designated in Fig. 1 as $B$. caesiocinereum group) contained sequences of specimens morphologically identical to the type material of $B$. caesiocinereum (smooth-spored) and B. caesiocinereum var. trachysporum (warted-spored). According to our results, these taxa should be accepted as two separate species; as a consequence, B. caesiocinereum var. trachysporum is raised to the species level. Additionally, five more lineages were detected in the $B$. caesiocinereum group. These are introduced as new species below. Two of them (B. glaucum and $B$. robenae) are smooth-spored, two species ( $B$. inconspicuum and $B$. walleynii) have warted basidiospores, and in one species (B. widdringtoniae) the spore wall bears spines. The smaller subclade (labelled as Basidiodendron cinerellum group, Fig. 1) contained sequences of specimens identical to a lectotype of Bourdotia cinerella; the latter species is redescribed and combined in Basidiodendron below. Alongside $B$. cinerellum with warted spores, three new species (introduced as $B$. groningae, $B$. mexicanum and $B$. spinulosum) with spiny basidiospores were uncovered in the B. cinerellum group. Rather high variability of ITS sequences of some species (in particular, B. caesiocinereum s. str. and $B$. trachysporum) prompted us to investigate their identity with the use of an additional marker.

\section{ITS + nc LSU rDNA + TEF1 dataset}

The final alignment contained 1884 characters (including gaps). The overall topologies of the ML and BI trees were nearly identical and in a good correspondence with the ITS + LSU phylogeny (Fig. 2). Both B. caesiocinereum s. str. and $B$. trachysporum lineages are strongly supported and therefore interpreted by us as representing single species each.

\section{ITS dataset}

Twenty additional environmental ITS sequences related to $B$. caesiocinereum complex were retrieved from GenBank and UNITE and used in the phylogenetic analyses (Supplement). Seven of them belonged to B. trachysporum and confirmed this species is widespread in temperate-boreal forests of Eurasia and North America. Basidiodendron caesiocinereum, $B$. cinerellum and $B$. walleynii were represented by three, two and one environmental sequences, respectively. Seven remaining sequences potentially represent four more species in the B. caesiocinereum complex: two from Canada (British Columbia) (KP889384, KP889562), one from temperate Europe (Austria) (JF519252, JF519305) and possibly one more represented by sequences from UK, Estonia and Alaska (AF504871, KF297103, UDB0141409). However, we could not connect these sequences with available herbarium material, and therefore their identity remains unresolved.

The morphology-based species identification in the $B$. caesiocinereum complex mainly relies on the presence and character of basidiospore ornamentation. As stated under "Material and methods," phase contrast illumination and Cotton Blue as a mountant are compulsory for this investigation. Of twelve species treated below, three species have completely smooth basidiospores and in nine species they are ornamented (Figs. 3, 4). Among the latter ones, four species possess warted and five have spiny outgrowths on the spore wall. Other morphological traits should also be considered for a correct species recognition. In particular, three species with warted basidiospores occurring in Europe (B. cinerellum, B. trachysporum and B. walleynii) are distinguishable due to differently looking basidiocarps and gloeocystidia, as well as specific arrangement of hymenial cells. Differentiating characters of $B$. caesiocinereum and related species are summarized in Table 2. Sequenced collections are marked by asterisk.

\section{Taxonomy}

Basidiodendron caesiocinereum (Höhn. \& Litsch.) LuckAllen, Canadian J. Bot. 41: 1036, 1963. - Figs. 3, 4, 5 and 6 $\equiv$ Corticium caesiocinereum Höhn. \& Litsch., Sitzungsb. Kaiserl. Akad. Wissenschaften, Math.-Naturw. Klasse Abt. 1, 117: 1116, 1908. Holotype. Germany. Nordrhein-Westfalen: Steinfurt, Lengerich, rotten hardwood, 1908 Brinkmann (FH 00304795, studied).

Basidiocarps effused, smooth, first waxy, pruinose or somewhat gelatinized, semitranslucent, greyish, then compact, greyish-bluish, older basidiocarps with a faint ochraceous-brownish tint, $0.02-0.03(0.05) \mathrm{mm}$ thick, up $3 \mathrm{~cm}$ in widest dimension, margin gradually thinning-out. Hyphal 
\begin{tabular}{l|lll} 
& \\
smooth spores & MK098920/MK098964 Ofella glaira
\end{tabular}

warted spores

* spiny spores

\begin{tabular}{|c|c|}
\hline $1 / 99$ & MK098920/MK098964 Ofella glaira \\
\hline $1 / 100$ & MG735416/MG735424 Hyalodon antui \\
\hline $1 / 100$ & MG735414/MG735422 Hyalodon piceicola \\
\hline & MG757508 Protomerulius subreflexus \\
\hline & MK0988884/MK0989632 Hydrophana sphaerospora \\
\hline & MK098883/MK098931 Hydrophana sphaerospora \\
\hline
\end{tabular}

Hyalodon

Protomerulius

Hydrophana

Endoperplexa

Mycostilla

Pseudohydnum

1/100_AF384861 Pseudohydnum gelatinos

1/100 DK098923/MK098967 Protoacia delica

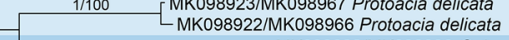
MK098882/MK098930 Gelacantha pura

Protoacia

Gelacantha

Ductifera

AY509551 Ductifera sucina

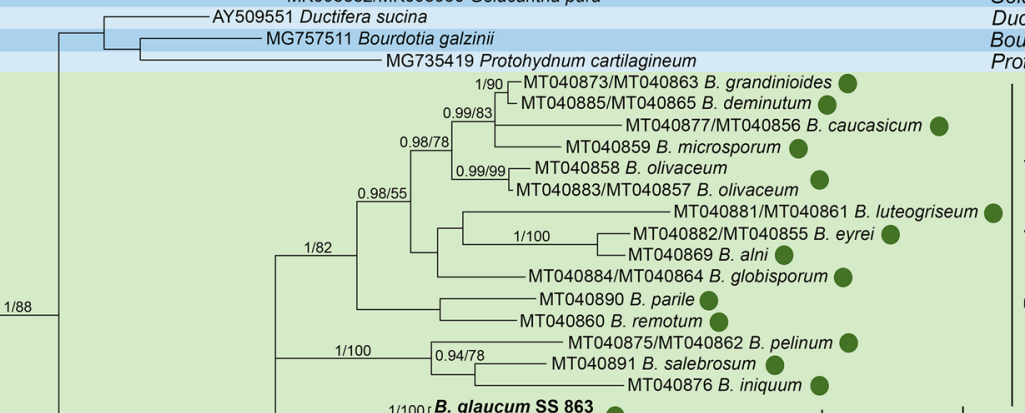

Protohydnum

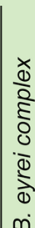

है

$1 / 100$

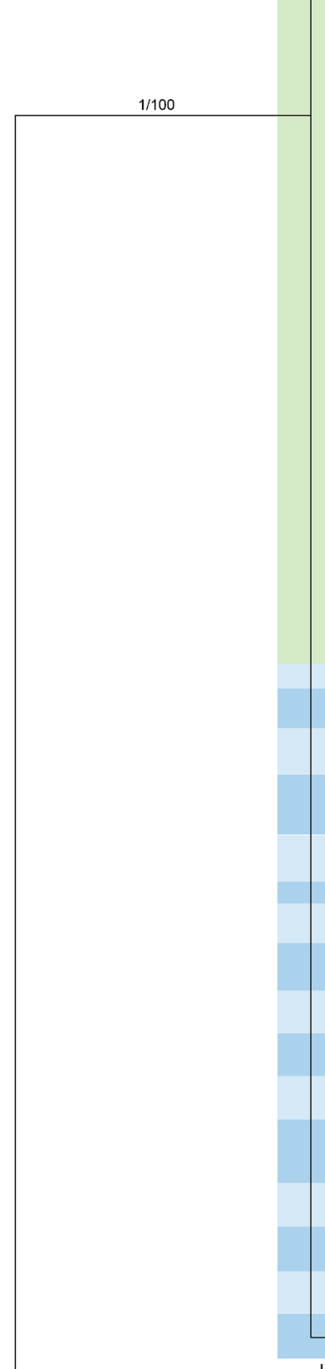

$0.96 / 46\left[\begin{array}{l}\text { B. caesiocinereum VS } 11115 \\ \text { B. caesiocinereum VS } 13663 \\ \text { B. caesiocinereum VS } 11776\end{array}\right.$

$1 / 99$ AF291293 B. caesiocinereum

B. robenae OM 16910.2

B. robenae OM 19650

$1 / 80 \quad$ B. widdringtoniae LR 11307a *
B. walleynii VS 9697

B. trachysporum VS $9188 \mathrm{a}$

$\left[\begin{array}{l}\text { B. trachysporum VS } 9483 \\ \text { B. trachysporum VS } 12508\end{array}\right.$

B. trachysporum VS 12528

B. trachysporum VS 9481

B. trachysporum VS 11803

B. trachysporum VS 11801

B. trachysporum VS 11111

B. trachysporum VS 12548

B. trachysporum VS 11888

$1 / 77$ B. trachysporum SS 608

B. trachysporum VS 8740

B. trachysporum HK 26387

B. trachysporum HK 29616

B. cinerellum VS 11188

B. cinerellum VS 11188

B. cinerellum VS 13485

$\begin{array}{ll}1 / 100 & \text { B. cinerellum VS } 13681 \\ \text { B. cinerellum VS } 13275\end{array}$

B. cinerellum VS 1327

B. spiculosum LR 23324 *

$1 / 100$

0.99/96 B. gron. spiculosum

0.99/96 B. groningae ENZ 18-001

B. groningae ENZ 19-073 *

DQ520100 Tremiscus helvelloides
$1 / 100 \quad$ MK098886/MK098934 Myxariellum tenerum

MK098978/MK098973 Protodontia africana

1/100 $\quad\left[\begin{array}{l}\text { MK098978/MK098973 Protodontia africana } \\ \text { MG735412/MG735420 Protodontia subgelatinosa }\end{array}\right.$

$1 / 100$

$1 / 93$

KY801883/KY801910 Myxarium populinum Y801882/KY801909 Myxarium cinnamomescens

1174 AF291281/AF291328 Exidiopsis grisea

$1 / 96 \sqrt{-}$ KX262110/KX262160 Tremellochaete japonica

$0.99 / 65 \quad$ KY801875/KY801900 Exidia candida

1/100 KY801872/KY801897 Exidia candida

$1 / 100 \quad K X 262140 / K X 262188$ Sclerotrema griseobrunneum

KX262144/KX262192 Sclerotrema griseobrunneum

$1 / 100-$ KX262135/KX262183 Heteroradulum kmetii

$1 / 99$ AF291271/AF291292 Auricularia mesenterca

AB915191 Auricularia mesenterica
$1 / 100$ JX044145 Aporpium caryae

esenterica

1/1100 [X044145 Aporpium caryae

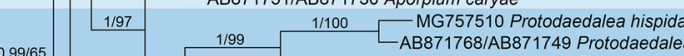

$1 / 87$ AB871768/AB871749 Protodaedalea hispida

MG757509 Elmerina cladophora

$0.9301 / 1 / 00+1 \times 262100$

0.93/61 1/100 KX262100/KX262147 Eichleriella crocata

1/98 KX262111/KX262161 Eichleriella leucophaea

1/100_MG857095/MG857099 Stypellopsis farlowi

MG857096/MG857102 Stypellopsis hyperborea

-JX535169/JX535170 Sistotrema brinkmannii 
४Fig. 1 Combined phylogenetic ITS + nc LSU rDNA topology from Bayesian analysis showing main lineages within the Auriculariales. Sequences generated for this study are indicated in bold. GenBank/ UNITE or collection numbers (for newly generated sequences) are given for all sequences. Support values (BS/PP) are given above the branches. Scale bar shows expected changes per site

structure monomitic, hyphae clamped; subicular hyphae thin- or slightly thick-walled, subparallel, (2) 3-4 (5.5) $\mu \mathrm{m}$ diam, subhymenial hyphae thin-walled, easily collapsing, ascending or interwoven, 2-3 (3.5) $\mu \mathrm{m}$ diam. Gloeocystidia rather abundant to rare, tapering, slightly projecting, hyaline to yellowish, (13) 14-40 (41) × (4) 4.2-9.4 (10.0) $\mu \mathrm{m}(n$ $=86 / 11)$. Basidia four-celled, ovoid to suburniform, (11.8) 12.2-24.2 (26.8) × (7.0) 7.2-11.4 (12.3) $\mu \mathrm{m}(\mathrm{n}=339 / 20)$, occasionally with a distinct stalk-like base up to $10 \times 3-5$ $\mu \mathrm{m}$, sterigmata gradually tapering, up to $15 \times 2.5-3 \mu \mathrm{m}$; involucres poorly developed. Basidiospores smooth, thinwalled, compressed-subglobose or globose, (5.0) 5.1-7.8 (8.3) $\times(5.2) 5.3-8.1(8.8) \mu \mathrm{m}(\mathrm{n}=780 / 26), \mathrm{L}=5.57-6.93$, $\mathrm{W}=5.97-7.28, \mathrm{Q}^{\prime}=(0.8) 0.9-1.0, \mathrm{Q}=0.93-1.00$, apiculus prominent, regular, sometimes slightly asymmetric, up to 2 $\times 2 \mu \mathrm{m}$.

Distribution and ecology. Europe (Belgium, Estonia, Finland, Germany, Italy, Norway), Asia (China, Russia - Siberia); almost exclusively on rotten wood of deciduous trees, as a rule in excessively humid habitats.

Remarks. Höhnel and Litschauer (1908) described $C$. caesiocinereum based on a single collection from Germany. They overlooked inner septation of basidia as well as the presence of cystidia, and this was a reason for them to assign the new species to the genus Corticium s. lato. Bourdot and Galzin (1927) restudied the type material of $C$. caesiocinereum and found that it has gloeocystidia and fourcelled basidia. They concluded that $C$. caesiocinereum is an older name for Bourdotia cinerella Bourdot \& Galzin. This viewpoint has persisted in the literature until the present day. However, we argue below that $B$. cinerella represents a warted-spored taxon, while the type specimen of $C$. caesiocinereum has smooth basidiospores. Therefore, the synonymy of $C$. caesiocinereum and $B$. cinerella should be abandoned.

As redefined here, $B$. caesiocinereum is most similar to $B$. glaucum. The two species are best separated by their ecological preferences. Basidiodendron glaucum is a northern species almost exclusively restricted to coniferous wood, while $B$. caesiocinereum is connected to angiosperm hosts, mainly in inundated habitats. However, B. glaucum may accidentally occur on deciduous trees, and one sequenced collection of $B$. caesiocinereum came from spruce. In these cases, $B$. caesiocinereum can be distinguished from B. glaucum due to longer basidia occasionally provided with a long stalk-like base. Basidiospores of $B$. caesiocinereum are on average larger than in $B$. glaucum, although their variation ranges are strongly overlapping. The distribution areas of both $B$. caesiocinereum and $B$. glaucum stretch along the northern part of Eurasia. No verified records of these species exist from North America. See B. robenae for further comments.

Specimens examined. Belgium. Vlaams-Brabant: Hoeilaart, Zoniënwoud, Kersselaerspleyn, Fagus sylvatica, X.2018 Schoutteten 18-172*, 18-226*, 18-625*, 18-902*, 18-925*, 18-1050*, 18-1051* (GENT). China. Jilin: Antu Co., Huang Song Pu, Populus sp. (?), 28.VIII.2005 Miettinen 10608* $(\mathrm{H})$. Finland. Varsinaissuomi: Bromarv, Rilax, deciduous wood, 13.X.2000 Saarenoksa 12100 (H). Uusimaa: Helsinki, Myllypuro - Puotinharju, Betula sp., 3.IX.1989 Saarenoksa 18789 (H), Vanhakaupunki, Alnus incana, 10.IX.1989 Saarenoksa 22889 (H), hardwood, 18.IX.1998 Saarenoksa 17598 (H), Veräjämäki, Salix caprea (?), 19-21.X.2011 Miettinen 14910.1, 14934.2 (H), Betula sp., 25.X.2019 Miettinen 22920.1 (H); Porvoo, Stensböle, S. caprea, 1.XI.1990 Kotiranta $9337(\mathrm{H})$; Sipoo, Lilla Kummelberget Nat. Res., Populus tremula, 29.IX.2010 Kotiranta 22735 (H). Enontekiön Lappi: Enontekiö, Kilpisjärvi, Betula pubescens spp. tortuosa, 2.IX.1983 Kotiranta $4745 a(\mathrm{H})$. Germany. Bavaria: Bad Hindelang, Hinterstein, strongly decayed deciduous wood, 20.IX.1995 Weiß 1995-320* (M.W.). Italy. Lombardy: Varese, Valganna, San Gemolo, Corylus avellana, 14.X.2019 Spirin 13663* (H). Norway. Vest-Agder: Lyngdal, Skoland, Ulmus glabra, 1.XI.2017 Spirin 11764* (O, H), rotten wood, 1.XI.2017 Larsson 17730, 17733, 17735, 17737, 17751 (O); Mandal, Uføra, Betula pubescens, 2.XI.2017 Spirin 11776*(O), C. avellana, 2.XI.2017 Spirin 11780 (O). Aust-Agder: Grimstad, Sæveli, C. avellana, 2.XI.2017 Spirin 11788 (O). Vestfold: Larvik, Jordstøyp i Kvelde, Tilia cordata, 30.IX.2018 Spirin 12536*(O), U. glabra, 30.IX.2018 Spirin 12538, 12539, 12542 (O), Vemannsås, U. glabra, 30.IX.2018 Spirin 12523 (O). Telemark: Bamble, Rognsheia, A. incana, 3.XI.2017 Spirin 11799 (O); Nome, Mørkvasslia, A. incana, 25.X.2016 Spirin 11187 (O), Picea abies, 16.X.2011 Svantesson 901* (O F253623). Buskerud: Lier, Asdøljuvet, A. incana, 29.IX.2018 Spirin 12515*(O), C. avellana, 29.IX.2018 Spirin 12500*(O), U. glabra, 29.IX.2018 Spirin $12502 *, 12504 *, 12511 *(\mathrm{O})$. Akershus: Asker, Esvika, Acer platanoides, 28.IX.2018 Spirin 12462 (O), U. glabra, 28.IX.2018 Spirin 12465*(O); Baerum, Kjaglidalen, A. incana, 16.IX.2016 Spirin 11115*, $11121(\mathrm{O})$, C. avellana, 16.IX.2016 Spirin 11123, 11126 (O), S. caprea, 16.IX.2016 Spirin 11129 (O). Oppland: Nord-Fron, Liadalane, A. incana, 12.IX.2016 Spirin 11044 (O), 29.IX.2017 Spirin 11636, 11649 (O), rotten wood, 29.IX.2017 Larsson 17631 (O); Sel, Sagåa, S. caprea, 13.IX.2016 Spirin 11069 (O). Møre og Romsdal: Nesset, Eikesdalen, A. incana, 27.IX.2017 Spirin 11610 (O), rotten wood, 27-28.IX.2017 Larsson 17548, 17558 (O). Russia. Krasnoyarsk Reg.: Turukhansk Dist., Lebed', Alnus hirsuta, 23.VIII.2013 Kotiranta 26428*(H). 
Fig. 2 Combined phylogenetic ITS + nc LSU rDNA + $T E F 1$ topology from Bayesian analysis showing phylogenetic relationships of the Basidiodendron caesiocinereum complex. GenBank/UNITE or collection numbers (for newly generated sequences) are given for all sequences. Support values (BS/ Scale bar shows expected changes per site $\mathrm{PP})$ are given on the branches.

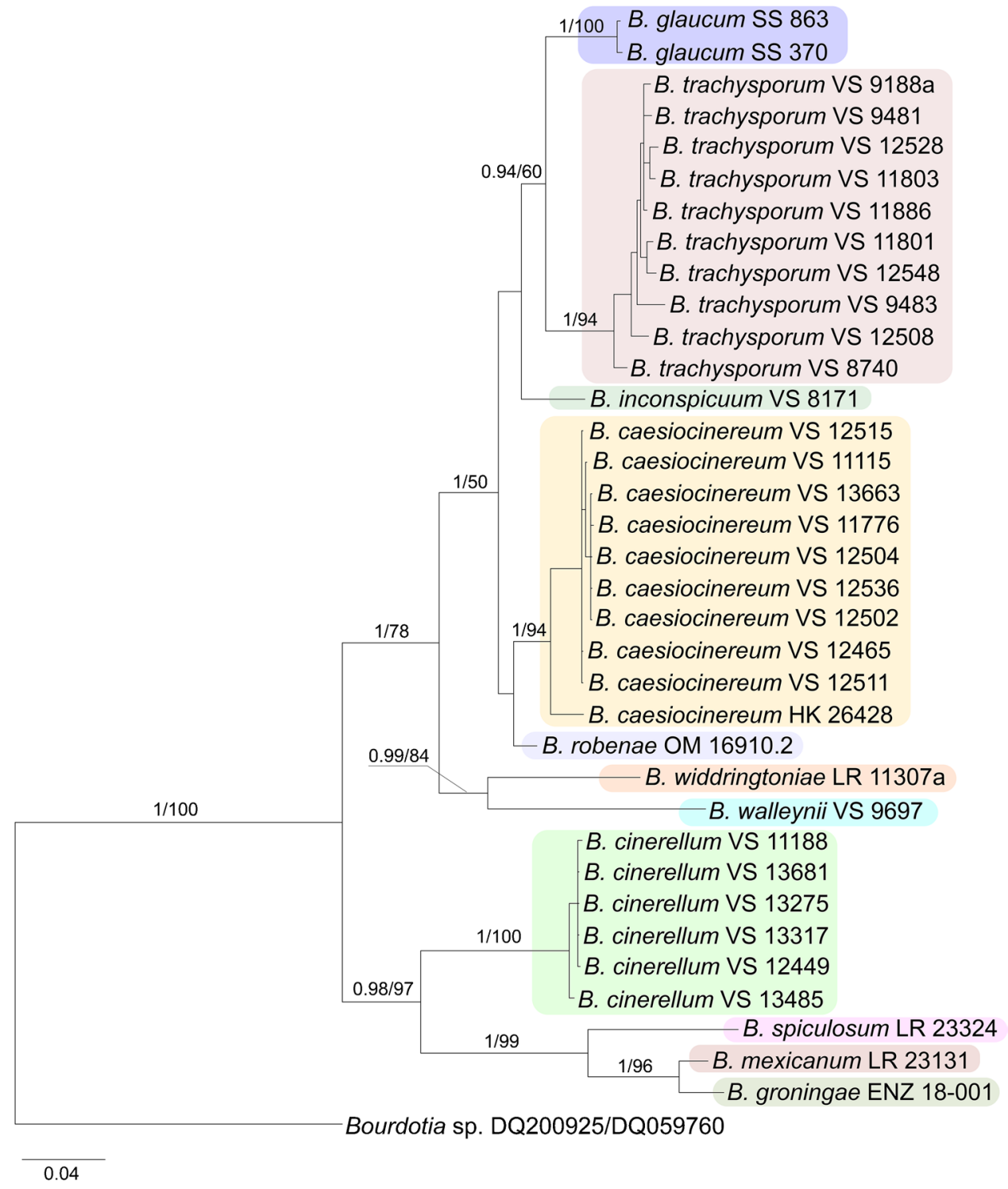

Basidiodendron cinerellum (Bourdot \& Galzin) Spirin \& V. Malysheva, comb. nov. - Figs. 3, 4, 5 and 7 MB 838719

$\equiv$ Bourdotia cinerella Bourdot \& Galzin, Bull. Soc. Mycol. France 36: 71, 1920. Lectotype (selected here, MBT395945). France. Aveyron: Causse Noir, Pinus sp., 20.XI.1913 Galzin 14526 (herb. Bourdot 12419) (PC 0706677).

Basidiocarps effused, smooth, first waxy, pruinosereticulate, greyish, then gelatinized, continuous, dirty-grey to pale ochraceous or brownish, occasionally tuberculate, often with irregularly scattered craters, $0.01-0.05 \mathrm{~mm}$ thick, covering a few $\mathrm{cm}$, margin gradually thinning-out. Hyphal structure monomitic, hyphae clamped, glued together; subicular hyphae thin-walled, subparallel, 2.5-4 $\mu \mathrm{m}$ diam, subhymenial hyphae thin-walled, ascending or interwoven, occasionally twisted, 1.5-3 $\mu \mathrm{m}$ diam, basidia-bearing hyphae distinct in older parts of hymenium, slightly thickwalled, up to $12 \times 2-3.5 \mu \mathrm{m}$. Gloeocystidia abundant, more or less clearly tapering, slightly projecting, hyaline or yellowish to brownish, (13.5) 14-34 (35) $\times$ (3.4) 3.7-8.0 (9.0) $\mu \mathrm{m}(n=75 / 8)$, solitary or in groups of $2-5$. Basidia four-celled, ovoid to broadly suburniform, (8.8) 8.9-16.0 $(17.0) \times(6.3) 7.0-10.2(10.3) \mu \mathrm{m}(n=70 / 7)$, sterigmata gradually tapering, up to $10 \times 1.5-2 \mu \mathrm{m}$; involucres welldeveloped, often totally covering basidial cells (except sterigmata); basidia in mature specimens embedded in gelatinous matrix and glued together in large groups. Basidiospores warted, thin- or slightly thick-walled (wall up to $0.2 \mu \mathrm{m}$ thick), compressed-subglobose or globose, (4.8-) 4.9-7.2 (-7.6) × (5.1-) 5.2-7.7 (-7.8) $\mu \mathrm{m}(\mathrm{n}=$ $330 / 11), \mathrm{L}=5.48-6.43, \mathrm{~W}=5.85-6.77, \mathrm{Q}^{\prime}=0.9-1.0, \mathrm{Q}=$ $0.94-0.97$, apiculus prominent, regular, sometimes slightly asymmetric, up to $1.5 \times 1.2 \mu \mathrm{m}$. 
Fig. 3 Microscopic structures of Basidiodendron spp. as seen in scanning electron microscope. a, b: B. caesiocinereum (holotype of Corticium caesiocinereum), basidium and basidiospores; c: B. cinerellum (lectotype of Bourdotia cinerella), basidiospores; d: B. groningae (holotype), basidiospores; e: $B$. spiculosum (holotype), basidiospores; f: $B$. spinosum (holotype of Sebacina spinosa), basidiospores
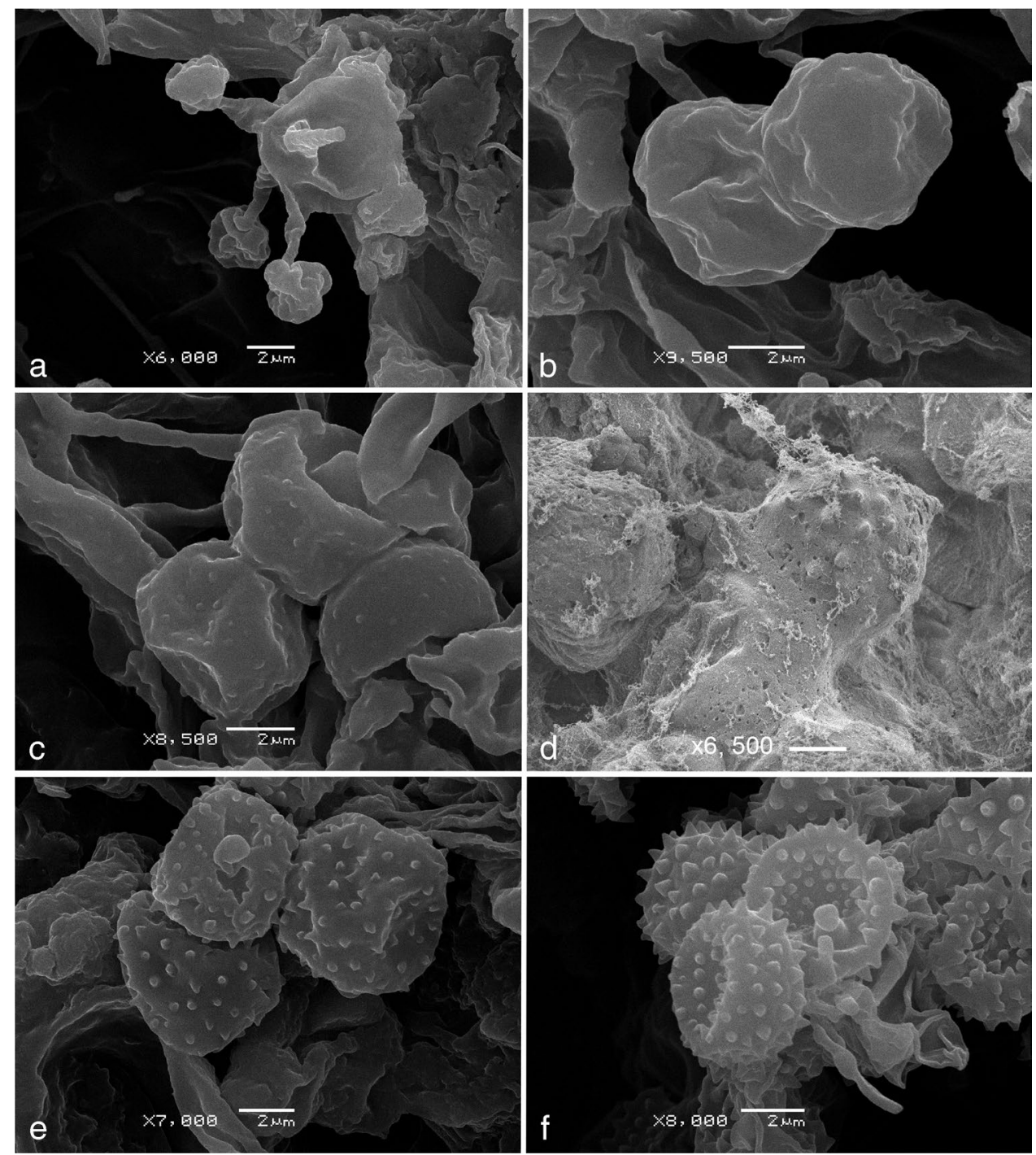

Distribution and ecology. Europe (Belgium, Estonia, Finland, France, Germany, Italy, Norway, North-West Russia, Slovenia, Sweden); on tough, often still corticated branches and fallen logs in various stages of decay, predominantly of conifers.

Remarks. Bourdot and Maire (1920) introduced $B$. cinerella from a large set of specimens collected in the southern part of France. They described it as having smooth spherical basidiospores and later considered it a synonym of $C$. caesiocinereum. However, B. cinerella has remained untypified, and therefore its actual relation to C. caesiocinereum was obscure. We studied all (in total 29) specimens stored in Bourdot's herbarium (PC), which were labelled by him as 'Sebacina (Bourdotia) cinerella'. Of them, two collections (Bourdot 40882 and 9047) belong to B. cinereum (Bres.) Luck-Allen s. lato, a species with cylindrical-ellipsoid spores; they do not fit to the protologue and cannot be used for typification. The rest of the specimens have globose spores, in good accordance with the original description, but they are warted, not smooth. Among them, two specimens represent Bourdotia cinerella var. trachyspora described seven years later (Bourdot and Galzin 1927) and considered here as a separate species. They certainly were not the main source for the $B$. cinerella description. Twelve remaining specimens were collected from coniferous (Pinus) and thirteen were from angiosperm hosts. However, both deciduous trees and conifers were mentioned as substrates in the protologue of $B$. cinerella, and therefore the host indication alone is insufficient for understanding the original idea of the species. Fortunately, Bourdot and Galzin provided a good description of macroscopic traits of $B$. cinerella: basidiocarps were described as 'whitish, whitish-grey, often glancing, pale ochraceous and crustaceous when old' ('blanchâtre, blanc-gris souvent brillant, subocracé et crustacé sur le vieux' - Bourdot and Galzin 1920: 71). These indications fit the pine-dwelling specimens and preclude collections from 
a<smiles></smiles><smiles></smiles>
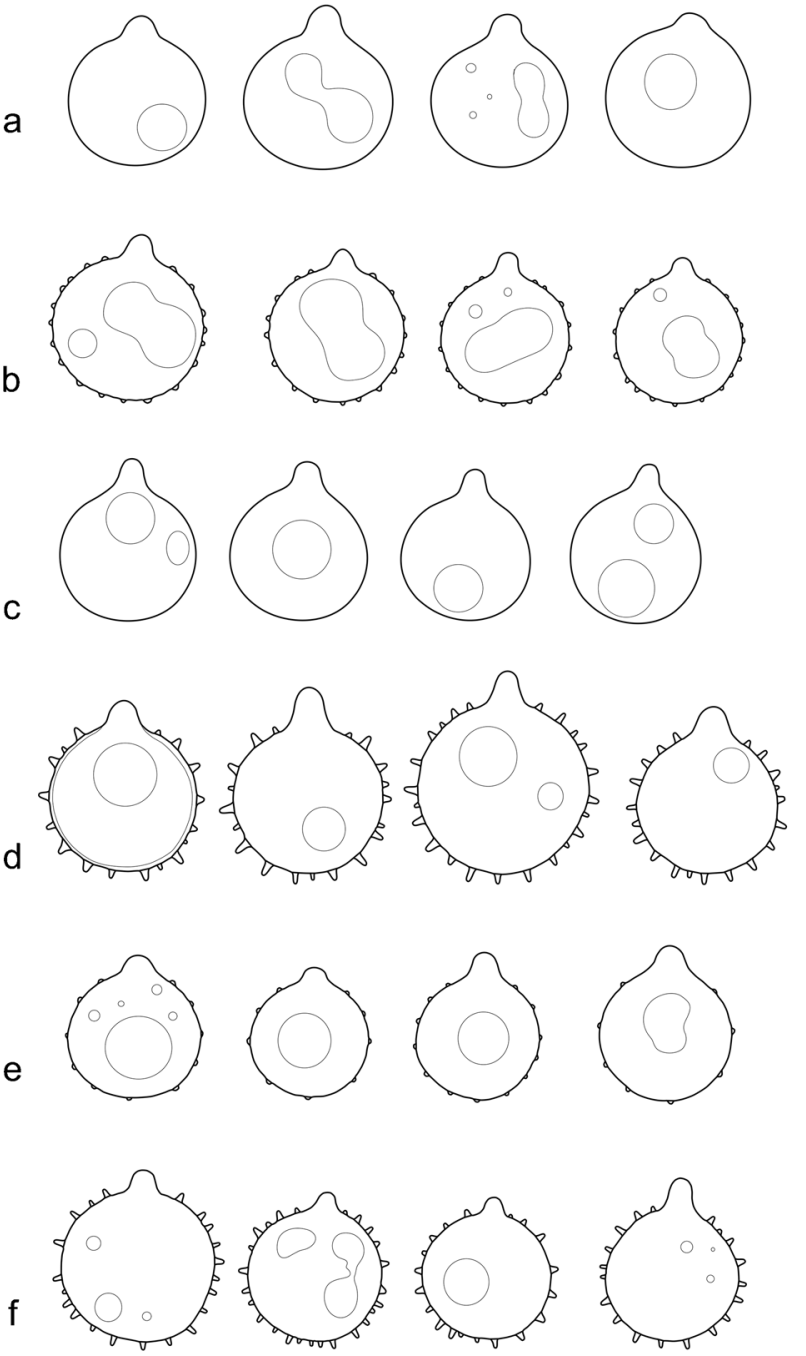

Fig. 4 Basidiospores of Basidiodendron spp. a: B. caesiocinereum (holotype of $C$. caesiocinereum); b: B. cinerellum (lectotype of Bourdotia cinerella); c: $B$. glaucum (holotype); d: $B$. groningae (holotype); e: $B$. incospicuum (holotype); f: $B$. mexicanum (holotype); g: $B$.

angiosperm hosts with arid, opaque basidiocarps. We assign the latter ones to a new species $B$. walleynii and select the best-developed specimen from Pinus (Galzin 14526, herb. Bourdot 12419) as a lectotype of B. cinerella.

Basidiodendron cinerellum is one of three species with warted basidiospores distributed in Europe. The most striking microscopic feature, differentiating $B$. cinerellum from two other species (i.e., B. trachysporum and B. walleynii), is the presence of a cyanophilous gelatinous matter covering basidial cells. In juvenile specimens, this matter is visible at least in some basidia as an essential $(1-2 \mu \mathrm{m})$ thickening of the basidial wall. In mature, and especially in senescent basidiocarps, the gelatinous matrix covers basidia up to the very top and glues them together in large, easily detectable groups. Moreover, basidiocarps of B. cinerellum often have dirty-greyish or ochraceous-brownish tints and, at least in
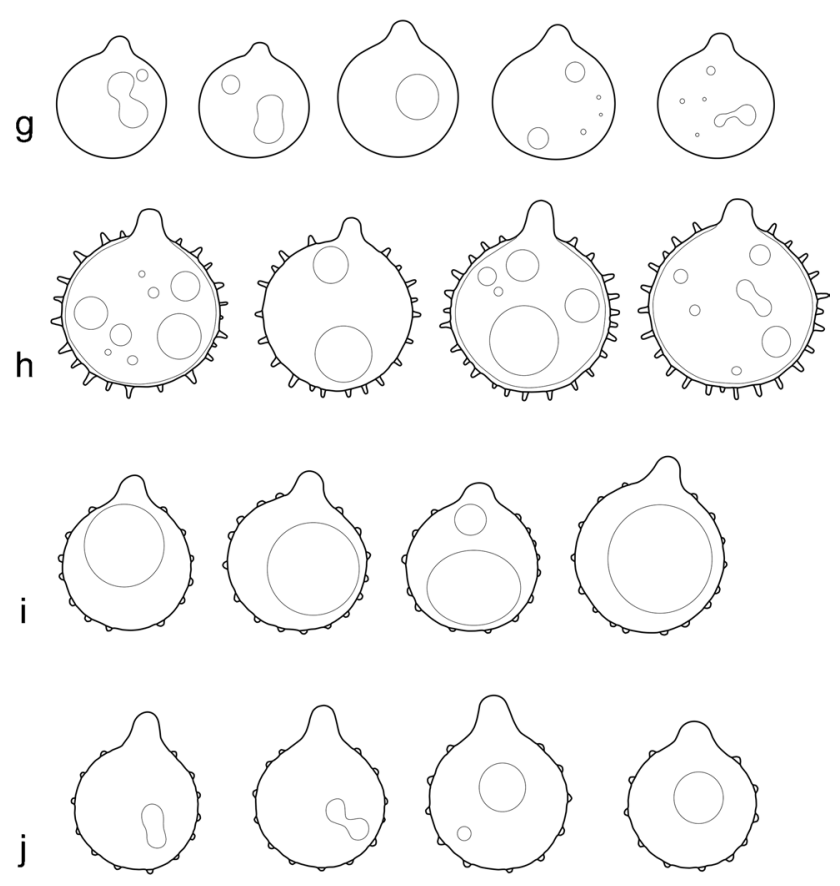

$\mathrm{k}$
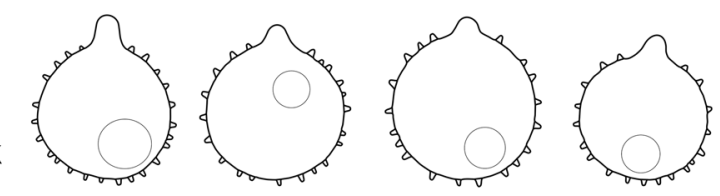

robenae (holotype); h: B. spiculosum (holotype); i: B. trachysporum (Spirin 12548); j: B. walleynii (holotype); k: B. widdringtoniae (holotype). Scale bar $=10 \mu \mathrm{m}$

some parts, they are more or less clearly gelatinized. In contrast, basidiocarps of $B$. walleynii remain arid and normally pale, while in B. trachysporum they are usually very thin, constantly whitish-greyish and occasionally gelatinized only when old. No signs of hymenial gelatinous matter so characteristic for B. cinerellum were detected in B. trachysporum or $B$. walleynii.

Basidiodendron cinerellum and B. trachysporum inhabit mostly coniferous hosts and sometimes occur in the same habitats. Our data suggest, however, that they may have quite different ecological specialization. Basidiodendron cinerellum seemingly prefers tough, often still corticated wood-mainly thick, still hanging or just fallen branches or small- to medium-sized logs. In turn, Basidiodendron trachysporum mostly occurs on well-decomposed wood, often on rotten logs lying on the ground or inside old stumps, 
Table 2 Geographic, ecological and morphological traits in Basidiodendron caesiocinereum complex

\begin{tabular}{|c|c|c|c|c|}
\hline Species & Geographic distribution & Host & Basidia & Basidiospores \\
\hline B. caesiocinereum & $\begin{array}{l}\text { Eurasia, temperate - hemibo- } \\
\text { real }\end{array}$ & Angiosperms & $12-24 \times 7-11.5 \mu \mathrm{m}$, exposed & $\begin{array}{l}\text { smooth, } \\
5.1-7.8 \times 5.3-8.1 \mu \mathrm{m}, \\
Q=0.93-1.00\end{array}$ \\
\hline B. cinerellum & Europe, temperate - boreal & $\begin{array}{l}\text { Mostly } \\
\text { gymnosperms }\end{array}$ & $\begin{array}{l}\text { 9-16 } \times 7-10 \mu \mathrm{m}, \text { glued in } \\
\text { groups }\end{array}$ & $\begin{array}{l}\text { warted, } \\
4.9-7.2 \times 5.2-7.7 \mu \mathrm{m}, \\
Q=0.94-0.97\end{array}$ \\
\hline B. glaucum & Eurasia, boreal & $\begin{array}{l}\text { Gymnosperms (predominantly } \\
\text { Picea) }\end{array}$ & $\begin{array}{l}10-14.5 \times 7.5-10.5 \mu \mathrm{m}, \\
\text { exposed }\end{array}$ & $\begin{array}{l}\text { smooth, } \\
5.1-6.8 \times 5.2-7.0 \mu \mathrm{m}, \\
Q=0.96-0.98\end{array}$ \\
\hline B. groningae & Europe, temperate & $\begin{array}{l}\text { Gymnosperms and angio- } \\
\text { sperms }\end{array}$ & $13-18 \times 9-12 \mu \mathrm{m}$, exposed & $\begin{array}{l}\text { spiny, } \\
6.0-7.9 \times 6.2-8.2 \mu \mathrm{m}, \\
Q=0.95-0.96\end{array}$ \\
\hline B. inconspicuum & $\begin{array}{l}\text { North America (North West), } \\
\text { temperate }\end{array}$ & Gymnosperms (Thuja) & $10-13 \times 7-9 \mu \mathrm{m}$, exposed & $\begin{array}{l}\text { warted, } \\
5.0-6.2 \times 5.2-6.5 \mu \mathrm{m}, \\
Q=0.95\end{array}$ \\
\hline B. mexicanum & North America, temperate & Gymnosperms (Pinus) & $12-15.5 \times 9.5-12 \mu \mathrm{m}$, exposed & $\begin{array}{l}\text { spiny, } \\
5.9-7.3 \times 6.1-7.4 \mu \mathrm{m}, \\
Q=0.96\end{array}$ \\
\hline B. robenae & North America, temperate & Angiosperms & $12-18 \times 7-9 \mu \mathrm{m}$, exposed & $\begin{array}{l}\text { smooth, } \\
5.1-6.4 \times 5.2-6.9 \mu \mathrm{m}, \\
Q=0.94-0.96\end{array}$ \\
\hline B. spiculosum & North America, subtropical & Fern remains & $15-24 \times 10-12 \mu \mathrm{m}$, exposed & $\begin{array}{l}\text { spiny, } \\
6.9-8.2 \times 7.1-8.9 \mu \mathrm{m}, \\
Q=0.96\end{array}$ \\
\hline B. spinosum & Oceania (Tahiti), tropical & Rotten wood & $9-10 \times 6-7.5 \mu \mathrm{m}$, exposed & $\begin{array}{l}\text { spiny, } \\
4.6-5.2 \times 4.8-5.8 \mu \mathrm{m} \text {, } \\
Q=0.96\end{array}$ \\
\hline B. trachysporum & $\begin{array}{l}\text { Eurasia and North America } \\
\text { (North-West), temperate - } \\
\text { boreal }\end{array}$ & Mostly gymnosperms & $8.5-16 \times 7-11 \mu \mathrm{m}$, exposed & $\begin{array}{l}\text { warted, } \\
4.8-7.4 \times 5.0-7.8 \mu \mathrm{m}, \\
Q=0.94-0.98\end{array}$ \\
\hline B. walleynii & Europe, temperate & $\begin{array}{l}\text { Angiosperms (mostly Castanea } \\
\text { and Quercus) }\end{array}$ & $11-15.5 \times 7-11 \mu \mathrm{m}$, exposed & $\begin{array}{l}\text { warted, } \\
5.1-7.0 \times 5.3-7.2 \mu \mathrm{m}, \\
Q=0.96-0.98\end{array}$ \\
\hline B. widdringtoniae & South Africa, subtropical & Gymnosperms (Widdringtonia) & $\begin{array}{l}11.5-14 \times 8-11 \mu \mathrm{m}, \text { glued in } \\
\text { groups }\end{array}$ & $\begin{array}{l}\text { spiny, } \\
5.9-6.8 \times 6.1-7.1 \mu \mathrm{m}, \\
Q=0.96\end{array}$ \\
\hline
\end{tabular}

although several records were made from small corticated coniferous branches.

Specimens examined. Belgium. Namur: Philippeville, Viroinval, Fondry des Chiens, Pinus sylvestris, 11.X.2019 Spirin 13485*, 13494 (H). Estonia. Viljandimaa: Tipu, Kikepera, P. sylvestris, 16.IX.2018 Spirin 12337* (H, TU114809), Lemmjõe, C. avellana, 17.IX.2018 Spirin 12350* (H, TU114820). Finland. Varsinaissuomi: Lohja, Lohjansaari, P. abies, 28.VIII.2003 Kotiranta 19980 (H); Tammisaari, Tenhola, P. abies, 1.IX.2004 Kotiranta 20450 (H). Uusimaa: Helsinki, Kumpula, P. abies, 16.IX.2001 Saarenoksa 04201 (H), Mölylä, P. abies, 4.XI.2017 Miettinen 21459 (H), Veräjämäki, P. abies, 30.X.2019 Viner 2019195 (H), P. sylvestris, 3.VI.2019 Spirin 12543 (H); Sipoo: Rörstrand, P. abies, 22.IX.2009 Miettinen 14005 (H); Vantaa, Vestra, dead Fomitopsis pinicola on P. abies, 10.V.2014 Spirin $6850(\mathrm{H})$. Etelä-Häme: Hämeenlinna, Ahvenaistenjärvi, $P$. abies, 22.IX.2016 Miettinen 20402 (H), Kotinen, P. abies, 25.IX.2014 Spirin 8099 (H). Etelä-Karjala: Virolahti, Klamila, decorticated board (old house), 5.VI.2010 Kotiranta $22704 a$ (H). Inarin Lappi: Utsjoki, Kevo, Juniperus communis, 20.IX.2009 Kotiranta 23123 (H). France. Aveyron: Causse Noir, Pinus sp., 3.XI.1911 Galzin 10147 (herb. Bourdot 9059) (PC 0706674), Galzin 10190 (herb. Bourdot 9061) (PC 0084210), 20.XI.1913 Galzin 14514 (herb. Bourdot 13962) (PC 0706665), Galzin 14520 (herb. Bourdot 13963) (PC 0706676), Galzin 14537 (herb. Bourdot 13964) (PC 0706673), 30.XI.1913 Galzin 14775 (herb. Bourdot 13966) (PC 0706675), 17.V.1915 Galzin 17703 (herb. Bourdot 20187) (PC 0706678), 5.XII.1915 Galzin 18879 (herb. Bourdot 15695) (PC 0706666), Galzin 18906 (herb. Bourdot 18566) (PC 0706660), Galzin 18919 (herb. Bourdot 15733) (PC 0706681), 16.V.1919 Galzin 24592 (herb. Bourdot 26851) (PC 0706682). Germany. Bavaria: Wertach, Jungholz, decayed $P$. 
Fig. 5 Basidiocarps of Basidiodendron spp. a: B. caesiocinereum (Spirin 11764); b: $B$. cinerellum (Spirin 13317); c: $B$. trachysporum (Spirin 11801); d: B. walleynii (Bourdot 19447). Scale bar $=1 \mathrm{~cm}$
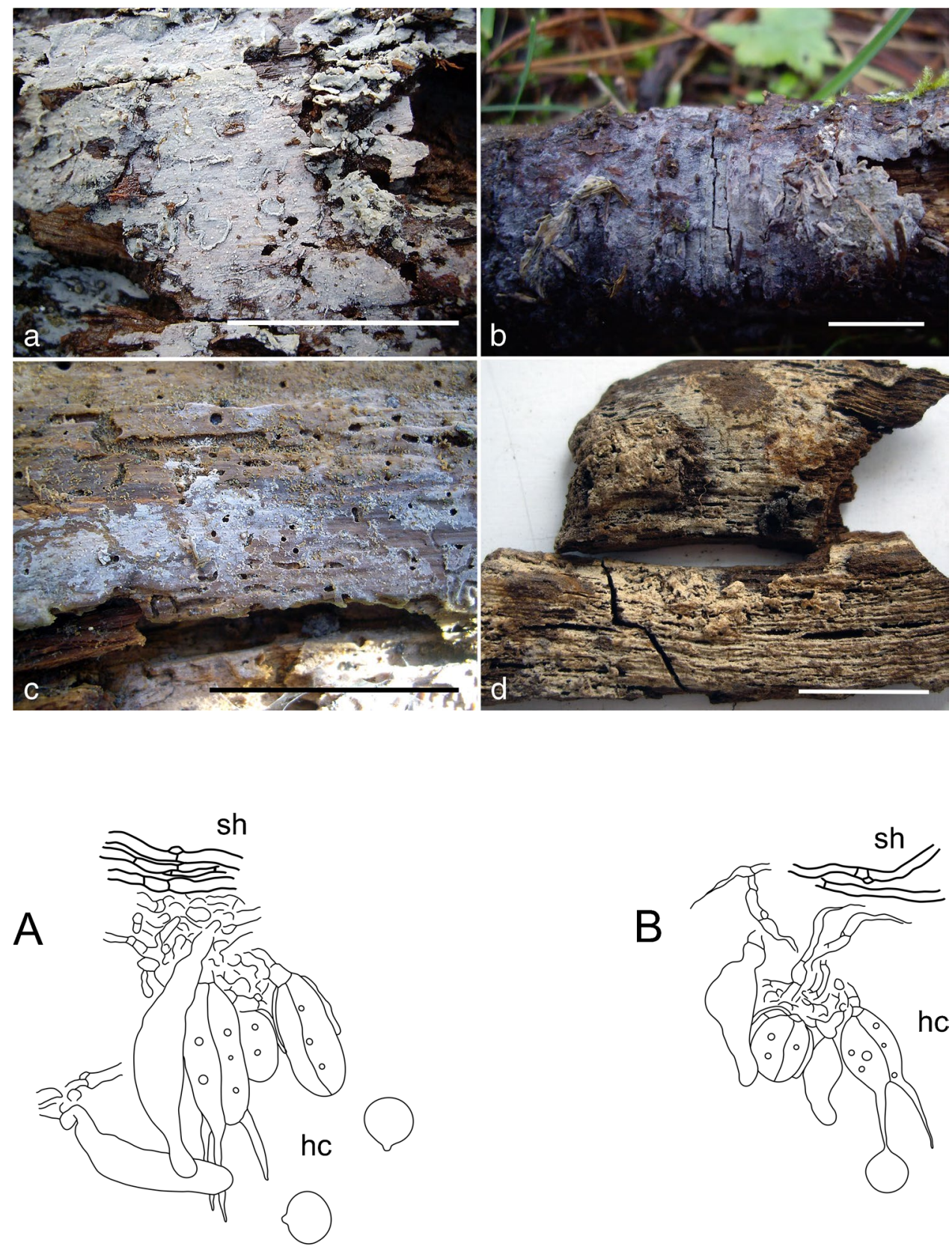

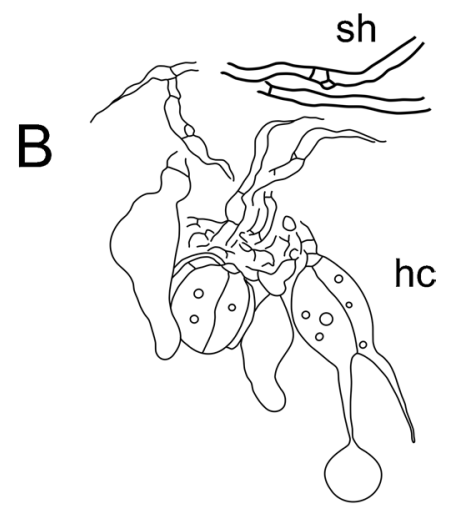

Fig. 6 Microscopic structures of smooth-spored Basidiodendron spp. A: B. caesiocinereum (holotype of $C$. caesiocinereum), B: B. glaucum (holotype), C: $B$. robenae (holotype); hc - hymenial cells, sh - subicular hyphae. Scale bar $=10 \mu \mathrm{m}$

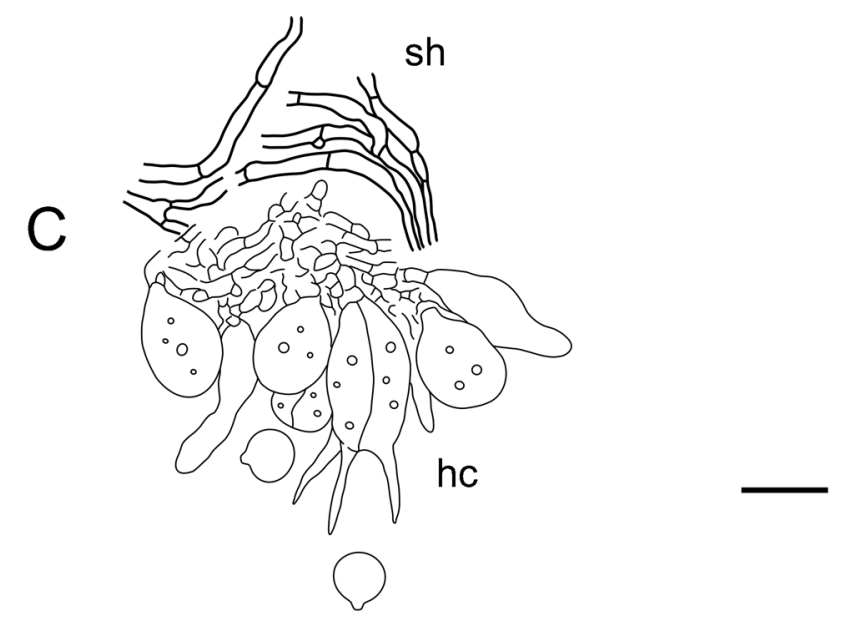



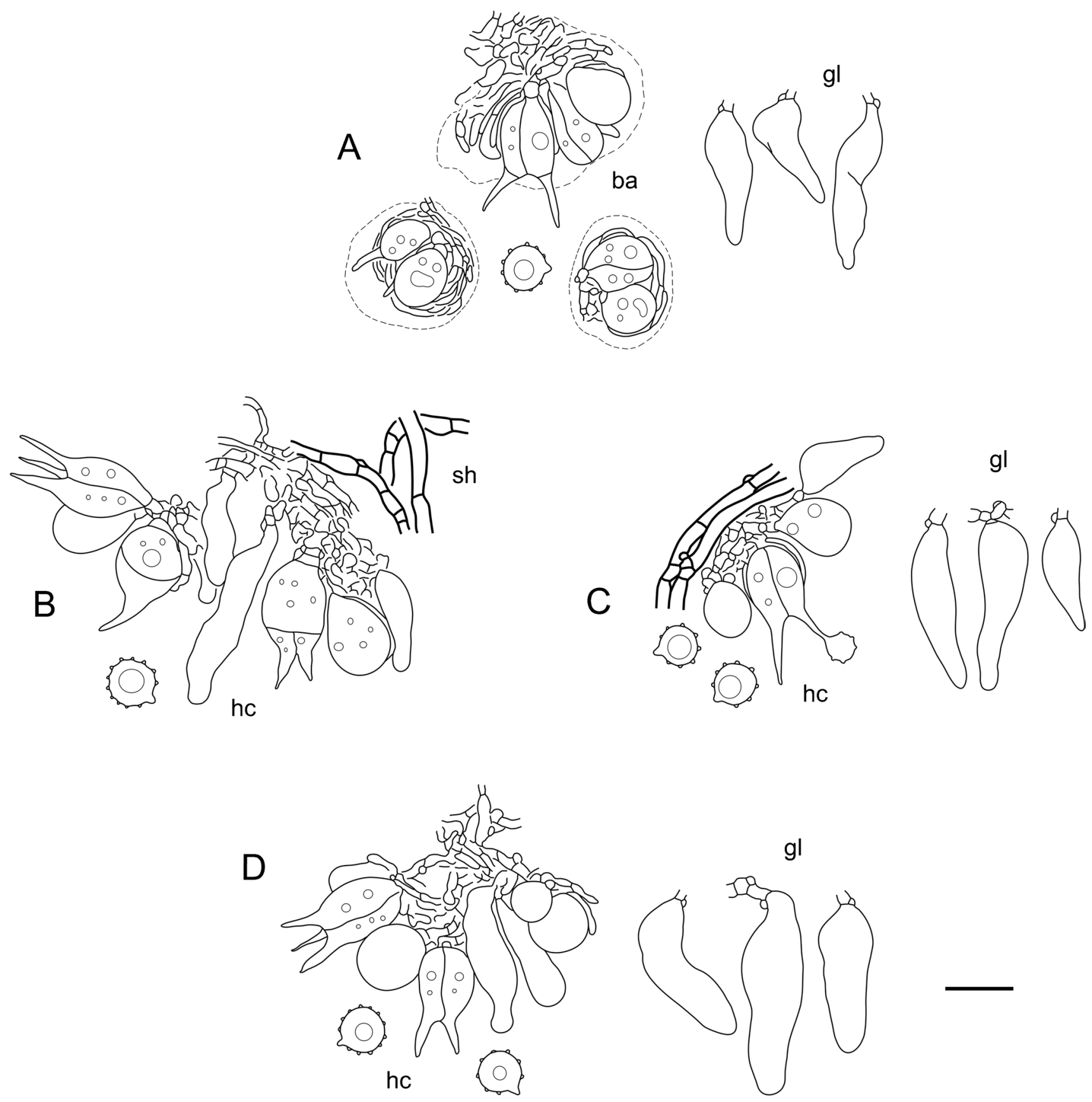

Fig. 7 Microscopic structures of European Basidiodendron spp. with ornamented spores. A: B. cinerellum (Spirin 12337), B: B. groningae (holotype), C: B. trachysporum (Spirin 11111), D: B. walleynii

(hymenial cells from holotype, gloeocystidia from Walleyn 3081); ba - basidia embedded in gelatinous matrix, gl - gloeocystidia, hc hymenial cells, sh - subicular hyphae. Scale bar $=10 \mu \mathrm{m}$

abies, 8.X.1996 Weiß 1996-393* (M.W.). Italy. Lombardy: Varese, Bedero Valcuvia, Marteghetta, P. abies, 14.X.2019 Spirin $13681^{*}(\mathrm{H})$, P. sylvestris, 14.X.2019 Spirin $13692(\mathrm{H})$. Norway. Telemark: Bamble, Rognsheia, P. abies and dead Phellinus ferrugineofuscus, 3.XI.2017 Larsson 17829 (O); Nome, Mørkvasslia, A. incana, 25.X.2016 Spirin 11188* (O). Akershus: Asker, Stokkerelva at Åstaddammen, $U$. glabra, 28.IX.2018 Spirin 12449*(O); Baerum, Kjaglidalen,
P. abies, 4.V.2016 Spirin 10019 (O). Oppland: Lunner, Rinilhaugen Nat. Res., P. abies, 17.IX.2016 Spirin 11144 (O); Sel, Sagåa Nat. Res., P. abies, 13.IX.2016 Spirin 11059 (O). Russia. Leningrad Reg.: Boksitogorsk Dist., Anisimovo, P. abies, 15.VII.2014 Spirin 7004 (H), Vozhani, P. abies, 2.X.2016 Spirin 11168 (H); Kirishi Dist., Klinkovo, P. abies, 7.VIII.2019 Spirin 12872 (H); Podporozhie Dist., Vazhinka, P. abies, 16.IX.2017 Spirin 11405 (H). Yamalo-Nenets 
Autonomous Dist.: Priuralsky Dist., Sob', Picea obovata, 11.VIII.1969 Parmasto (TAAM 053842). Slovenia. Gorenjska: Kranjska Gora, Vršič, Pinus mugo, 5.VI.2019 Spirin 12576 (H), 27.IX.2019 Spirin 13317*, 13325, 13333, 13338, 13344 (H); Bohinj, Lipanca, L. decidua, 26.IX.2019 Spirin 13290, 13303 (H), 29.VII.2020 Spirin 13945 (H), P. abies, 6.VI.2019 Spirin 12594, 12613, 12626 (H), 26.IX.2019 Spirin 13229, 13237 (H), P. mugo, 26.IX.2019 Spirin 13275*, 13281, 13287, 13293 (H), Mrežce, P. abies, 26.IX.2019 Spirin 13221 (H), Mrzli Studenec, P. abies, 27.VII.2020 Spirin 13879 (H), Ravne v Bohinju, Abies alba, 28.VII.2020 Spirin 13888 (H), Studor, P. abies, 27.VII.2020 Spirin 13852 (H); Mojstrana, Triglavska Bistrica, P. abies, 28.IX.2019 Spirin 13418, 13422, $13471(\mathrm{H})$. Sweden. Kalmar: Vimmerby, Norra Kvill, P. abies, 27.X.2010 J. Nordén 7879 (O). Örebro: Lekeberg, Ugglehöjden, P. abies, 13.X.2010 J. Nordén 7561 (O).

Basidiodendron glaucum Spirin \& K.H. Larss., sp. nov. - Figs. 4 and 6

MB 838720

Holotype. Norway. Nord-Trøndelag: Snåsa, Blåfjella, 64.2939N 13.029E, Picea abies (decorticated log), 28.IX.2011 J. Nordén 9683* (O F-248006, isotype - H).

Etymology: Glaucus (Lat., adj.) - bluish-grey, in reference to the basidiocarp's colour.

Basidiocarps effused, smooth, first waxy, pruinose, then continuous, arid, greyish, 0.02-0.06 (0.1) mm thick, up 6 $\mathrm{cm}$ in widest dimension, margin gradually thinning-out. Hyphal structure monomitic, hyphae clamped; subicular hyphae thin-walled, subparallel, 2-3 $\mu \mathrm{m}$ diam, subhymenial hyphae thin-walled, easily collapsing, ascending or interwoven, 1.5-2 (2.5) $\mu \mathrm{m}$ diam. Gloeocystidia rather rare, tapering, slightly projecting, hyaline to yellowish, (14) 14.5-34.0 $(38.0) \times(3.7) 4.0-8.2(9.0) \mu \mathrm{m}(\mathrm{n}=30 / 3)$. Basidia fourcelled, ovoid to broadly suburniform, (10.1) 10.2-14.3 $(14.8) \times(7.0) 7.7-10.3(11.0) \mu \mathrm{m}(\mathrm{n}=62 / 6)$, sterigmata gradually tapering, up to $13 \times 2.5-3 \mu \mathrm{m}$; involucres poorly developed. Basidiospores smooth, thin-walled, compressedsubglobose or globose, (5.0) 5.1-6.8 (7.0) $\times(5.0) 5.2-7.0$ (7.2) $\mu \mathrm{m}(\mathrm{n}=270 / 9), \mathrm{L}=5.53-6.24, \mathrm{~W}=5.67-6.37, \mathrm{Q}^{\prime}=$ 0.9-1.0 (1.1), Q = 0.96-0.98, apiculus prominent, usually slightly asymmetric, up to $2 \times 1.8 \mu \mathrm{m}$.

Distribution and ecology. Europe (France, Norway, North-West Russia, Sweden), Asia (Russian Far East); almost exclusively on rotten wood of conifers.

Remarks. Basidiodendron glaucum is distributed in borealsubalpine coniferous forests of Eurasia. It seems to occur mostly in old-growth communities with abundant wood remnants. Basidiodendron globisporum Spirin \& V. Malysheva has the same ecological preferences. It can be distinguished from B. glaucum by the smaller basidia $(9-12 \times 7-9 \mu \mathrm{m})$ and basidiospores $(4.6-5.8 \times 4.8-5.9 \mu \mathrm{m}, \mathrm{L}=4.95-5.15, \mathrm{~W}=$
5.10-5.33) with a less prominent apiculus (Spirin et al. 2020). Differences between B. glaucum and the look-alike B. caesiocinereum are listed above. Basidiodendron robenae is morphologically almost indistinguishable from B. glaucum but it was detected only on angiosperm hosts in North America.

Specimens examined (paratypes). France. Vosges: SaintDié-des-Vosges, Plainfaing, Col du Bonhomme, A. alba, 12.X.2019 Spirin 13539 (H). Norway. Vest-Agder: Lyngdal, Fladstad, A. incana, 1.XI.2017 Spirin 11750*(O). Akershus: Nannestad, Rudskampen, P. abies, 10.X.2011 J. Nordén 9815* (O F-248007). Telemark: Drangedal, P. abies, 18.X.2011 J. Nordén 9920* (O F253676); Nome, Mørkvasslia, P. abies, 16.X.2011 J. Nordén 9858* (O F253666); Sandalslia, $P$. abies, 13.X.2011 Svantesson 863*(O). Akershus: Nannestad, 8.X.2011 J. Nordén 9760 (O). Sør-Trøndelag: Selbu, Råndalen, P. abies, 19-20.IX.2011 J. Nordén 9313, 9326, 9339 (O), Svantesson 351 (O); Tydal, Hilmo, P. abies, 22.IX.2011 Svantesson 370*(O), 23.IX.2011 J. Nordén 9573 (O). NordTrøndelag: Snåsa, Blåfjella, P. abies, 27.IX.2011 J. Nordén 9605 (O), 28.IX.2011 J. Nordén 9678, 9680 (O). Nordland: Hattfjelldal, Nordlia, P. abies, 9.IX.2011 J. Nordén 9079* (O F253604), J. Nordén 9080* (O F253638); Grane, Litltuva, P. abies, 5-6.IX.2011 Svantesson $10(\mathrm{O})$, Svantesson $11 *(\mathrm{O}$ F253609), Svantesson 52 (O), Svantesson 140*(O F253677), Svantesson 141, 144* (O). Russia. Khabarovsk Reg.: Verkhnebureinskii Dist., Dublikan Nat. Res., Picea ajanensis, 23.VIII.2014 Spirin 7890* (H 7028622). Leningrad Reg.: Kirishi Dist., Shariya, P. abies, 10.VIII.2019 Spirin 12922 (H). Sweden. Västra Götaland: Strömstad, Hästeskede, $P$. abies, 31.X.2011 J. Nordén 10037 (O). Västerbotten: Lycksele, Altarliden, P. abies, 29.IX.2010 J. Nordén 7152 (O).

Basidiodendron groningae Schoutteten \& Spirin, sp. nov. - Figs. 3, 4 and 7

MB 838721

Holotype. Netherlands. Groningen: Lauwersoog, Ballastplaatbos, 53.402N 6.213E, Hippophae rhamnoides (dry corticated branch), 10.XI.2018 Schoutteten 18-1325* (GENT, isotype $-\mathrm{H})$.

Etymology: Groninga (Lat., noun) - a Latin name of Groningen, the type locality.

Basidiocarps effused, smooth, first waxy, pruinosereticulate, greyish, then continuous, greyish to pale ochraceous, 0.02-0.04 mm thick, covering a few mm, in some portions slightly gelatinized, margin gradually thinning-out. Hyphal structure monomitic, hyphae clamped; subicular hyphae thin- or slightly thick-walled, interwoven, 2-3 $\mu \mathrm{m}$ diam, subhymenial hyphae thin- to slightly thick-walled, ascending or interwoven, 1.5-2 $\mu \mathrm{m}$ diam. Gloeocystidia abundant, distinctly tapering, slightly projecting, hyaline to yellowish, (14) 18-34 (41) $\times$ (4.0) 4.1-7.0 (7.5) $\mu \mathrm{m}(n=$ $20 / 1)$, solitary or in groups of 2-7. Hyphidia not observed. 
Basidia normally four-celled, ovoid to broadly suburniform, (12.8) 12.9-17.8 (18.2) × (8.4) 9.0-12.0 (12.2) $\mu \mathrm{m}(n=$ $30 / 2$ ), sterigmata gradually tapering, up to $14 \times 2.5-3$ $\mu \mathrm{m}$; some basidia obliquely or even transversally septate, 1-2-sterigmatic, occasionally pleural; involucres indistinct. Basidiospores spiny (spines up to $0.3-0.6 \mu \mathrm{m}$ long), thinwalled or with slightly thickened (up to $0.3 \mu \mathrm{m}$ ) walls, compressed-subglobose or globose, (5.9) 6-7.9 (9.1) $\times(6.1)$ 6.2-8.2 (9.4) $\mu \mathrm{m}(\mathrm{n}=90 / 3), \mathrm{L}=6.35-7.14, \mathrm{~W}=6.68-7.40$, $\mathrm{Q}^{\prime}=0.9-1.0, \mathrm{Q}=0.95-0.96$, apiculus prominent, regular or eccentric, sometimes slightly asymmetric, up to $3 \times 1.8 \mu \mathrm{m}$.

Distribution and ecology. Europe (Belgium, Netherlands); dead wood of unidentified conifers and deciduous trees (Hippophae rhamnoides).

Remarks. Basidiodendron groningae is the only species with spiny basidiospores so far detected in Europe. It was collected three times around Groningen in the Netherlands and once in Belgium, but its actual distribution is unknown. Four other species with spiny spores dealt with in this paper were found in North America (B. mexicanum, B. spiculosum), Africa (B. widdringtoniae) and Oceania (B. spinosum). In the type specimen of $B$. groningae, basidia occasionally bear oblique or even transversal septa, and then only one or two apical cells produce sterigmata. This feature illustrates morphological flexibility of basidial cells most likely caused by environmental conditions and seems to have no taxonomic importance.

Specimens examined (paratypes). Belgium. Antwerpen: Boeckhout, Den Turck, rotten wood, 6.II.2020 Van Autgaerden 20-040* (GENT). Netherlands. Groningen: Lauwersoogbos, fallen corticated coniferous branch, 11.XI.2018 Enzlin 18-001* (GENT); Oude Pekela, Pekelderbos, fallen corticated coniferous branch, 24.X.2019 Enzlin 19-073* (GENT).

Basidiodendron inconspicuum Spirin \& V. Malysheva, sp. nov. - Figs. 4 and 8

MB 838722

Holotype. USA. Washington: Clallam Co., Willoughby Creek, 47.8218N 124.1983W, Thuja plicata (very rotten decorticated log), 7.X.2014 Spirin 8171* (H, isotype - LE).

Etymology: Inconspicuus (Lat., adj.) - inconspicuous, in reference to the hardly detectable basidiocarps of the species.

Basidiocarps effused, smooth, waxy, pruinose-reticulate, whitish or greyish, 0.01-0.02 mm thick, covering a few $\mathrm{mm}$, hardly detectable. Hyphal structure monomitic, hyphae clamped; subicular hyphae slightly thick-walled, subparallel, 2-3 $\mu \mathrm{m}$ diam, subhymenial hyphae thin- or slightly thickwalled, ascending or interwoven, 1.5-3 $\mu \mathrm{m}$ diam. Gloeocystidia abundant, tapering, slightly projecting, hyaline to yellowish, (12) 13-27 (37) × (3.6) 3.8-7.8 (8.3) $\mu \mathrm{m}(n=20 / 1)$, solitary. Basidia four-celled, ovoid to broadly suburniform, (9.8) $10.2-13.2(13.7) \times(6.8) 7.0-8.8(9.2) \mu \mathrm{m}(n=20 / 1)$, sterigmata gradually tapering, up to $8 \times 1.5 \mu \mathrm{m}$; involucres normally poorly developed, well-visible in senescent hymenium only. Basidiospores minutely warted, thin-walled, compressedsubglobose or globose, (4.8) 5.0-6.2 × (5.1) 5.2-6.5 (6.7) $\mu \mathrm{m}(\mathrm{n}$ $=30 / 1), \mathrm{L}=5.49, \mathrm{~W}=5.76, \mathrm{Q}^{\prime}=0.9-1.0, \mathrm{Q}=0.95$, apiculus regular, sometimes slightly asymmetric, up to $1.2 \times 1.5 \mu \mathrm{m}$.

Distribution and ecology. So far known from the type locality; strongly decayed wood of conifers (Thuja plicata).

Remarks. Basidiodendron inconspicuum produces very thin, hardly detectable basidiocarps consisting of two-three subparallel basal hyphae and sparse subhymenial hyphae bearing hymenial cells. Morphologically, it is most similar to B. trachysporum. The latter species has on average larger basidiospores with more pronounced (up to 0.2-0.3 $\mu \mathrm{m}$ long) warts. In turn, the basidiospore ornamentation of $B$. inconspicuum is seen only under phase contrast as tiny (0.1-0.2 $\mu \mathrm{m}$ long) warts regularly arranged on the spore surface. Phylogenetically, $B$. inconspicuum is more closely related to the smooth-spored $B$. caesiocinereum than to $B$. trachysporum.

Basidiodendron mexicanum Spirin \& V. Malysheva, sp. nov. - Figs. 4 and 8

MB 838723

Holotype. Mexico. Vera Cruz: Cofre de Perote, Mpio de Xico, El Revolcadera, 19.473N 97.154W, Pinus patula (rotten decorticated log), 18.IX.1985 Ryvarden 23131* (O, isotypes $-\mathrm{H}, \mathrm{LE}$ ).

Etymology: Mexicanus (Lat., adj.) - after Mexico, where the species was collected.

Basidiocarps effused, smooth, first waxy, pruinose, whitish or greyish, then continuous, greyish to pale ochraceous, arid, $0.02-0.03 \mathrm{~mm}$ thick, covering a few $\mathrm{cm}$, occasionally gelatinized and semitranslucent, margin gradually thinningout. Hyphal structure monomitic, hyphae clamped; subicular hyphae slightly thick-walled, subparallel, 2-3.5 $\mu \mathrm{m}$ diam, subhymenial hyphae slightly thick-walled, ascending or interwoven, frequently anastomosing, 2-3.5 $\mu \mathrm{m}$ diam. Gloeocystidia abundant, distinctly tapering, slightly projecting, hyaline to yellowish or rarely brownish, (11) 17-34 (35) $\times$ (4.2) 4.3-6.2 (7.0) $\mu \mathrm{m}(n=20 / 1)$, solitary or in groups of $2-3$. Hyphidia occasionally present, mostly simple, $1-1.5$ $\mu \mathrm{m}$ in diam. (apical part), projecting up to $15 \mu \mathrm{m}$. Basidia four-celled, ovoid to broadly suburniform, (11.5) 11.8-15.7 $(16.0) \times(9.2) 9.7-12.2(12.3) \mu \mathrm{m}(\mathrm{n}=20 / 1)$, sterigmata gradually tapering, up to $15 \times 2-2.5 \mu \mathrm{m}$; involucres welldeveloped, often totally covering basidial cells (except sterigmata). Basidiospores spiny (spines up to $0.3-0.4 \mu \mathrm{m}$ long), thin-walled, compressed-subglobose or globose, (5.8) 5.9-7.3 (7.6) $\times 6.1-7.4(7.8) \mu \mathrm{m}(\mathrm{n}=30 / 1), \mathrm{L}=6.45, \mathrm{~W}=$ $6.69, \mathrm{Q}^{\prime}=0.9-1.0, \mathrm{Q}=0.96$, apiculus prominent, regular, sometimes slightly asymmetric, up to $2 \times 1.8 \mu \mathrm{m}$. 

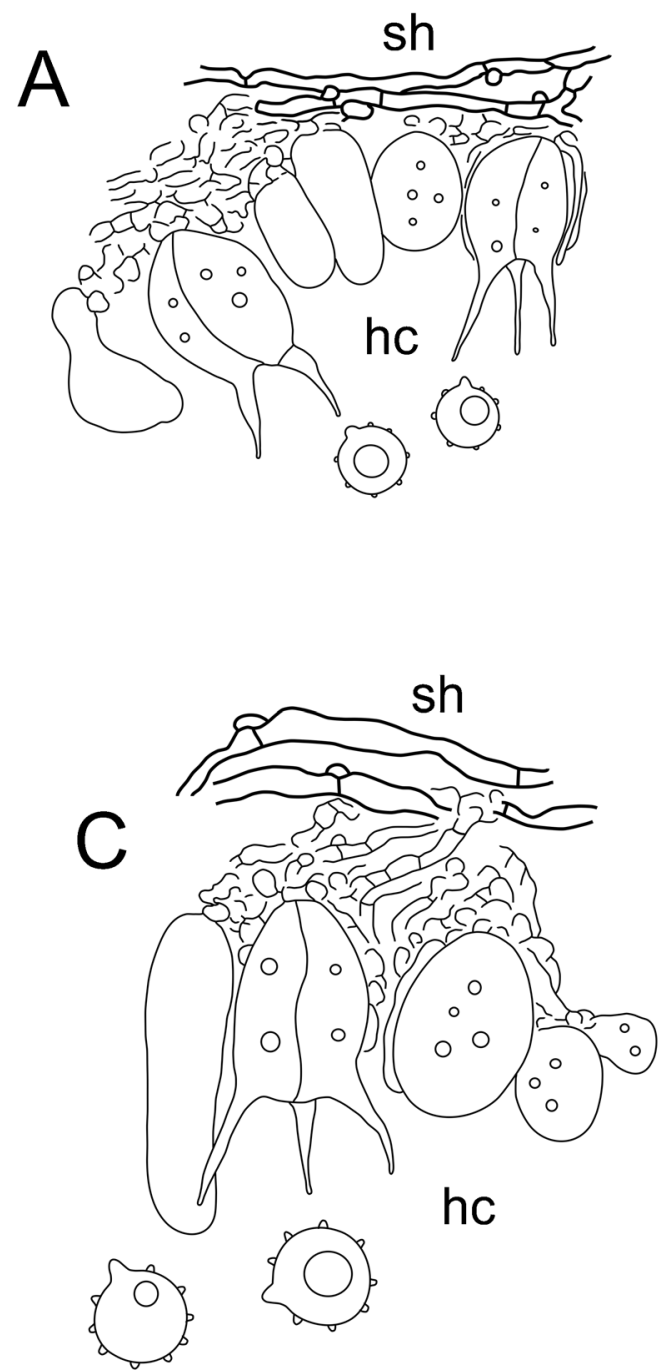

Fig. 8 Microscopic structures of extra-European Basidiodendron spp. with ornamented spores. A: B. inconspicuum (holotype), B: B. mexicanum (holotype), C: B. spiculosum (holotype), D: B. widdringtoniae

Distribution and ecology. So far known from the type locality; decorticated coniferous wood in a highland forest.

Remarks. Basidiodendron mexicanum is one of the species with spiny basidiospores introduced here. It differs from B. spiculosum, also found in Mexico, in having thinner basidiocarps, shorter gloeocystidia and smaller basidiospores, as well as by the presence of hyphidia. Phylogenetically, B. mexicanum is closely related to $B$. groningae so far detected only in Europe (Figs. 1, 2). The latter species possesses basidiospores with more pronounced spines than in B. mexicanum, and it lacks hyphidia. ITS sequences of these species show 2.7-3.9\% distance (the infraspecific differences within $B$. groningae are under 1.6\%). Morphologically, B. mexicanum is most similar to $B$. widdringtoniae (see remarks under the latter species). Basidiodendron widdringtoniae is so far known from Malawi only and phylogenetically closer to $B$. walleynii than to $B$. mexicanum or $B$. spiculosum.
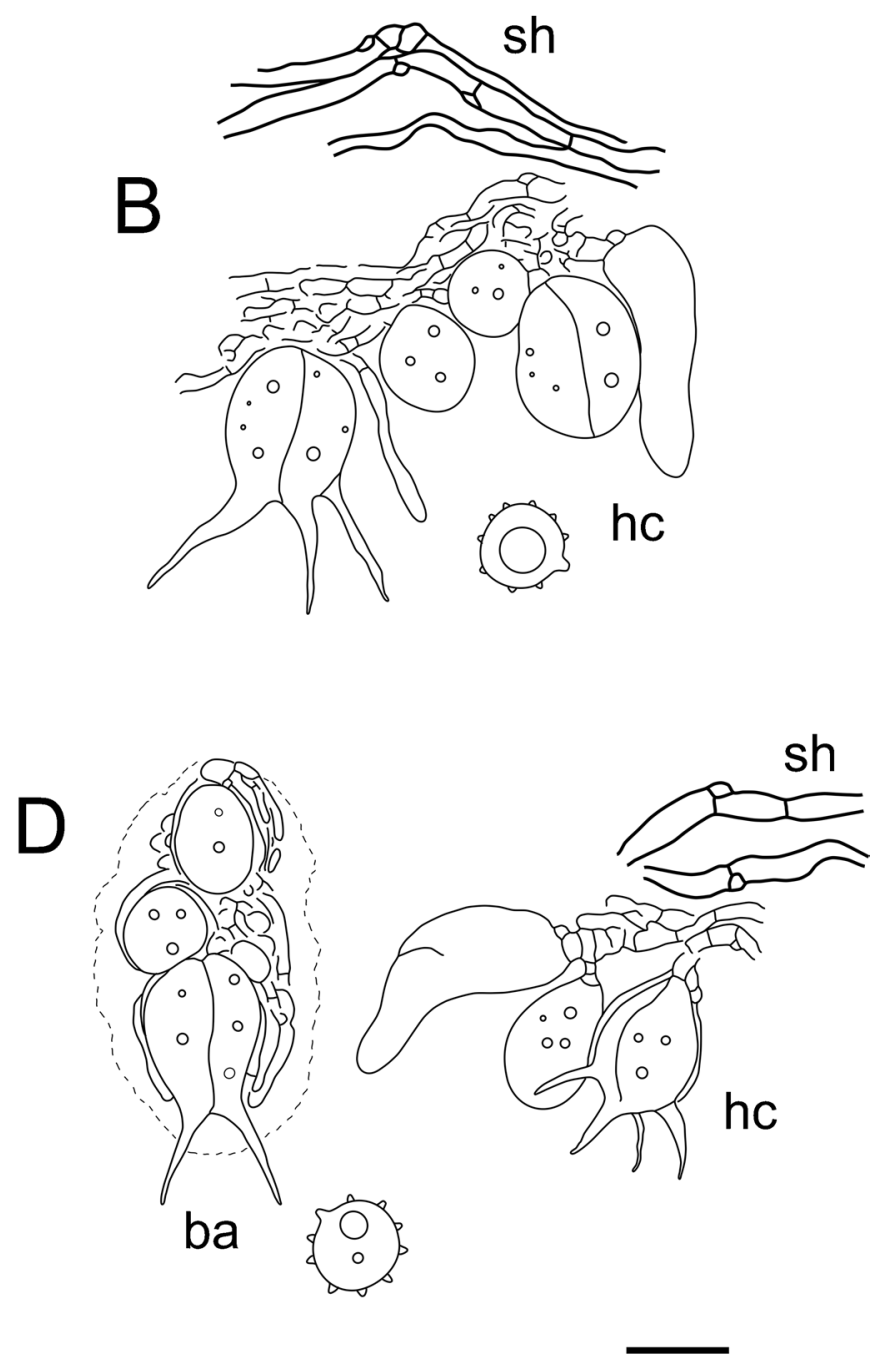

(holotype); ba - basidia embedded in gelatinous matrix, gl - gloeocystidia, hc - hymenial cells, sh - subicular hyphae. Scale bar $=10$ $\mu \mathrm{m}$

Basidiodendron robenae Spirin \& Miettinen, sp. nov. - Figs. 4 and 6

MB 838725

Holotype: USA, New York, Essex Co., Arbutus Lake, 43.9836N 74.2354W, fallen angiosperm tree, 16.IX.2013 Miettinen $16910.2 *(\mathrm{H})$.

Etymology: After Robena Luck-Allen, the first monographer of the genus Basidiodendron.

Basidiocarps effused, smooth, waxy, continuous, pale ochraceous to greyish, $0.02-0.05 \mathrm{~mm}$ thick, covering a few $\mathrm{cm}$ in widest dimension, margin gradually thinning-out. Hyphal structure monomitic, hyphae clamped; subicular hyphae slightly thickwalled, subparallel, occasionally glued together, 2-3.5 $\mu \mathrm{m}$ diam, subhymenial hyphae thin- to slightly thick-walled, glued together, ascending or interwoven, 2-3 $\mu \mathrm{m}$ diam, basidia-bearing hyphae distinct in older parts of hymenium, slightly thickwalled, up to $10 \times 2.5-3 \mu \mathrm{m}$. Gloeocystidia abundant, slightly or 
distinctly tapering, projecting, hyaline to yellowish, (15) 16-32 (33) $\times(5.2) 5.6-8.8(9.3) \mu \mathrm{m}(n=36 / 2)$. Basidia four-celled, ovoid to suburniform, (11.0) 12.2-18.2 (18.8) × (7.0) 7.1-9.2 (10.4) $\mu \mathrm{m}(n=40 / 2)$, sterigmata gradually tapering, up to $10 \times$ 1.5-2.5 $\mu \mathrm{m}$; involucres occurring in senescent hymenium, covering basidia up to the very top. Basidiospores smooth, thin- or slightly thick-walled, compressed-subglobose or globose, (5.0) $5.1-6.4 \times(5.1) 5.2-6.9(7.2) \mu \mathrm{m}(\mathrm{n}=90 / 3), \mathrm{L}=5.69-5.72, \mathrm{~W}$ $=5.95-6.03, \mathrm{Q}^{\prime}=0.9-1.0, \mathrm{Q}=0.94-0.96$, apiculus prominent, usually regular, up to $1.2 \times 1.8 \mu \mathrm{m}$.

Distribution and ecology. North America (Canada - Ontario, USA - New York, Tennessee); rotten wood of deciduous trees.

Remarks. Basidiodendron robenae is a smooth-spored species distributed in the north-eastern part of North America. It is morphologically almost indistinguishable from the Eurasian species $B$. glaucum. In addition to separated distribution areas, these species have different substrate preferences: $B$. robenae has been detected on angiosperm hosts while $B$. glaucum occurs almost exclusively on coniferous wood, mainly on Picea spp.

Specimens examined (paratypes). Canada. Ontario: York Co., Nashville, Fagus grandifolia, 22.X.1955 Cain (H ex TRTC 31661). USA. Tennessee: Cocke Co., Cosby Creek, fallen angiosperm tree, 2.X.2015 Miettinen 19650* $(\mathrm{H})$, Betula sp., 2.X.2015 Miettinen 19655 (H), Sevier Co., Ramsey Cascade Trail, Quercus sp., 30.IX.2015 Miettinen 19562 (H).

Basidiodendron spiculosum Spirin \& Ryvarden, sp. nov. - Figs. 3, 4 and 8

MB 838726

Holotype. Mexico. Vera Cruz: Xalapa, Botanical Garden, 19.514N 96.947W, Cyathea sp. (dry stem), 22.IX.1985 Ryvarden 23324* (O, isotypes - H, LE).

Etymology: Spiculosus (Lat., adj.) - spiculate, in reference to the basidiospore ornamentation.

Basidiocarps effused, smooth, waxy, pruinose-reticulate, whitish or cream-coloured, then pale ochraceous, $0.02-0.04 \mathrm{~mm}$ thick, covering a few $\mathrm{cm}$, margin gradually thinning-out. Hyphal structure monomitic, hyphae clamped; subicular hyphae slightly thick-walled, subparallel, 2-3 $\mu \mathrm{m}$ diam, subhymenial hyphae thin- or slightly thick-walled, interwoven, $1.5-2.5 \mu \mathrm{m}$ diam, quickly collapsing. Gloeocystidia abundant, tapering or tubularclavate, slightly projecting, hyaline to yellowish or brownish, (27) $28-51(55) \times(6.3) 6.8-10.0(11.3) \mu \mathrm{m}(\mathrm{n}=20 / 1)$, solitary of in groups of 2-3. Basidia four-celled, ovoid to broadly suburniform, (14.2) 14.8-24.2 (25.0) × (9.6) 9.8-12.0 (12.8) $\mu \mathrm{m}(\mathrm{n}=24 / 1)$, sterigmata gradually tapering, up to $10 \times 2-2.5 \mu \mathrm{m}$; involucres well-developed, often covering basidial cells up to the middle part. Basidiospores spiny (spines up to $0.3-0.4 \mu \mathrm{m}$ long), thinor slightly thick-walled (wall up to $0.3 \mu \mathrm{m}$ thick), compressedsubglobose or globose, (6.8) 6.9-8.2 (8.8) × (7.0) 7.1-8.9 (9.0) $\mu \mathrm{m}(n=30 / 1), \mathrm{L}=7.62, \mathrm{~W}=7.95$, Q' $=0.9-1.0, \mathrm{Q}=0.96$, apiculus prominent, regular, sometimes slightly asymmetric, up to $2 \times 2 \mu \mathrm{m}$.

Distribution and ecology. So far known from the type locality; dry stem of a tree fern.

Remarks. Of the species dealt with herein, B. spiculosum is most similar to $B$. mexicanum; their differences are listed under the latter species. It seems the diversity of the spinosespored Basidiodendron spp. in North America is not exhausted by two species only. Kisimova-Horovitz et al. (1997) reported B. spinosum from Costa Rica, and their description indicates the presence of one more, still unnamed species with spinose basidiospores.

Basidiodendron spinosum (L.S. Olive) Wojewoda, Mala Flora Grzybów 2: 91, 1981. - Fig. 3

三Sebacina spinosa L.S. Olive, Bull. Torrey Bot. Club 85: 27, 1958. Holotype. French Polynesia. Tahiti: Fautaua Valley, very rotten wood, 3.VII.1956 Olive T398 (NY 01293275, studied).

Basidiocarps effused, smooth, waxy, pruinose-reticulate, whitish or greyish, 0.03-0.05 mm thick, covering a few $\mathrm{cm}$, margin gradually thinning-out. Hyphal structure monomitic, hyphae clamped, densely arranged; subicular hyphae thin- to slightly thick-walled, subparallel, 1.5-2.5 $\mu \mathrm{m}$ diam, subhymenial hyphae thin- to slightly thick-walled, ascending or interwoven, $1.5-2.5 \mu \mathrm{m}$ diam. Gloeocystidia tapering or clavate, slightly projecting, hyaline or yellowish, $22-25 \times 5.5-10 \mu \mathrm{m}$. Basidia four-celled, ovoid to broadly suburniform, widely collapsed, ca. $9-10 \times 6-7.5 \mu \mathrm{m}$, sterigmata gradually tapering, up to $9 \times 2-2.5$ $\mu \mathrm{m}$; involucres indistinct. Basidiospores spiny (spines up to 0.6 $\mu \mathrm{m}$ long), thick-walled (wall up to $0.7 \mu \mathrm{m}$ thick), compressedsubglobose or globose, 4.6-5.2 $\times 4.8-5.8 \mu \mathrm{m}(\mathrm{n}=30 / 1), \mathrm{L}=$ $5.00, \mathrm{~W}=5.22, \mathrm{Q}^{\prime}=0.9-1.0, \mathrm{Q}=0.96$, apiculus prominent, regular, sometimes somewhat asymmetric, up to $1.2 \times 0.8 \mu \mathrm{m}$.

Distribution and ecology. So far known from the type locality; rotten wood of an unidentified tree.

Remarks. The only available material of this species is represented by the type specimen from Tahiti. Basidiodendron spinosum differs from other species of the $B$. caesiocinereum complex in having very prominent and densely arranged spines on the spore surface and clearly thick-walled basidiospores. Newly collected and sequenced specimens from the type locality are needed for clarifying phylogenetic relationships of $B$. spinosum with other representatives of the genus.

Basidiodendron trachysporum (Bourdot \& Galzin) Spirin, M. Weiß \& Miettinen, comb. nov. - Figs. 4, 5 and 7

MB 838727

$\equiv$ Bourdotia cinerella var. trachyspora Bourdot \& Galzin, Hyménomycètes de France: 50, 1927. Lectotype (selected here, MBT395946). France. Aveyron: Causse Noir, Pinus sp., 8.V.1911 Galzin 9106 (herb. Bourdot 9073) (PC). 
Basidiocarps effused, smooth or indistinctly tuberculate, first waxy, pruinose, whitish or greyish, then continuous, greyish to pale ochraceous, arid, $0.01-0.03 \mathrm{~mm}$ thick, covering a few $\mathrm{cm}$, occasionally gelatinized, semitranslucent and then almost invisible by a naked eye, margin gradually thinning-out. Hyphal structure monomitic, hyphae clamped; subicular hyphae thin- or slightly thick-walled, subparallel, (1.5) 2-3.5 $\mu \mathrm{m}$ diam, subhymenial hyphae thin- or slightly thick-walled, ascending or interwoven, 1.5-2.5 (-3) $\mu \mathrm{m}$ diam, basidia-bearing hyphae distinct in older parts of hymenium, slightly thick-walled, up to $15 \times 2.5-3 \mu \mathrm{m}$. Gloeocystidia abundant, tapering, slightly projecting, hyaline to yellowish, (12) 13-45 (60) $\times(2.8) 3.0-7.8(8) \mu \mathrm{m}(\mathrm{n}=129 / 15)$, solitary or in groups of 2-6. Basidia four-celled, ovoid to broadly suburniform, (8.2) 8.3-15.8 (16.0) × (6.7) 6.9-11.0 (11.2) $\mu \mathrm{m}(n=198 / 17)$, sterigmata gradually tapering, up to $10 \times$ 1.5-2.5 $\mu \mathrm{m}$; involucres normally poorly developed, wellvisible in senescent basidiocarps only. Basidiospores warted, thin-walled, compressed-subglobose or globose, 4.8-7.4 (7.8) $\times$ (4.9) 5.0-7.8 (7.9) $\mu \mathrm{m}(n=590 / 20), \mathrm{L}=5.26-6.58, \mathrm{~W}=$ 5.46-6.79, Q' = 0.9-1.0, Q = 0.94-0.98, apiculus prominent, regular, sometimes slightly asymmetric, up to $2 \times 1.8 \mu \mathrm{m}$.

Distribution and ecology. Europe (Estonia, Finland, France, Netherlands, Norway, Russia, Slovenia, Sweden, Ukraine), Asia (Russia - Siberia and Far East), North America (USA - Washington); predominantly strongly decayed wood, mostly of conifers.

Remarks. This species was initially introduced as a warted-spored variety of $B$. cinerella (Bourdot and Galzin 1927), while the latter was erroneously described as a smooth-spored species (see discussion under $B$. cinerellum). The single authentic specimen labelled by Bourdot as 'Sebacina (Bourdotia) cinerella f. trachyspora' is an extensive collection in good condition, and it is designated here as a lectotype. Morphological differences of B. trachysporum from B. cinerellum are listed under the latter species. Another similarly looking European species, B. walleynii, occurs on deciduous hosts and has thicker basidiocarps and differently shaped, wider and clearly projecting cystidia. Basidiodendron trachysporum is certainly the most common species of the genus in the middle- and north-boreal forests of North Europe.

The morphological variability of $B$. trachysporum deserves a closer look. In particular, one specimen collected in the North-American North-West (Spirin 8262) has unusually large basidia, $15-23 \times 11.5-14.5 \mu \mathrm{m}$, and basidiospores, (8.6) 9.0-11.2 (11.7) × (9.1) 9.2-11.9 (12.3) $\mu \mathrm{m}(\mathrm{n}=30 / 1), \mathrm{L}=9.69, \mathrm{~W}=10.00, \mathrm{Q}^{\prime}=0.9-1.0$ (1.1), $\mathrm{Q}=0.97$. However, the ITS sequence of this collection does not show any significant differences versus other ITS sequences of $B$. trachysporum, and our attempts to sequence additional markers were unsuccessful. For now, we treat the specimen under B. trachysporum.
Specimens examined. Estonia. Valgamaa: Otepää, Kääriku, decorticated decayed wood, 10.IX.2012 Põldmaa (TU 112986*), Välkjärve, $P$. tremula, 15.IX.2015 Spirin $9848(\mathrm{H})$. Finland. Ahvenanmaa: Geta, Getabergen, P. abies, 24.X.2001 Kotiranta 19148 (H), Snäkö, small wooden planks, 24.X.2001 Saarenoksa 18601 (H); Lumparland, Skag, J. communis, 23.X.2007 Kotiranta 22098 (H). Varsinaissuomi: Salo, Orjanperä, P. sylvestris, 10.V.2010 Kotiranta 22663 (H); Tammisaari, Tenhola, P. abies, 16.IX.2008 Kotiranta 22418 (H). Uusimaa: Helsinki, Kumpula, coniferous wood, 13.XI.1999 Saarenoksa 04699 (H), Patola, A. incana, 26.X.2011 Miettinen 14981.3 (H), Veräjämäki, P. abies, 26.IX.2018 Miettinen $21815(\mathrm{H})$, 1.XI.2019 Miettinen 22962.2* (H), P. sylvestris, 20.X.2019 Miettinen 22899, 22906 (H), Viikki, P. abies, 1.X.2000 Saarenoksa 07600 (H), 6.XI.2019 Viner 2019-215 (H); Inkoo, Sommarö Nat. Res., P. sylvestris (old collapsed house), 22.V.2010 Kotiranta 22695 (H); Kirkkonummi, Sundsberg, P. sylvestris, 17.V.2005 Kotiranta 21261 (H), 20.X.2012 Miettinen 15789.2 (H); Porvoo, Stensböle, P. abies, 1.XI.1990 Kotiranta 9354 (H). Etelä-Häme: Hämeenlinna, Kotinen, $P$. abies, 25.IX.2014 Spirin 8102 (H); Luhanka, Lempää, J. communis, 6.X.2010 Kotiranta 22796, 22805 (H); Padasjoki: Koivukannonsuo, P. abies, 6-7. IX.2003 Miettinen 7508.1 (H). France. Aveyron: L'Hospitalet, Pinus sp., 26.XI.1916 Galzin 21134 (herb. Bourdot 20188) (PC 0706680); St. Sernin, Cerasus sp. and debris, 3.VI.1909 Galzin 4250 (herb. Bourdot 20185) (PC 0706658). Netherlands. Groningen: Hoogezand, P. sylvestris, 7.II.2020 Enzlin 20-005* (GENT). Norway. Vestfold: Larvik, Jordstøyp i Kvelde, $P$. abies (old collapsed building), 15.IX.2016 Spirin 11111*(O), Vemannsås, P. abies, 30.IX.2018 Spirin 12528*, $12530(\mathrm{O})$. Telemark: Bamble, Rognsheia, P. abies, 3.XI.2017 Spirin 11801*, 11803*(O), rotten wood, 3.XI.2017 Larsson 17796 (O); Nome, Mørkvasslia, P. abies, 25.X.2016 Spirin 11196 (O). Akershus: Nannestad, P. abies, 8.X.2011 J. Nordén 9770 (O). Buskerud: Lier, Asdøljuvet, P. abies, 29.IX.2018 Spirin 12508* (O), Stokkerinden, P. abies, 29.IX.2018 Larsson 17910 (O). Hedmark: Åmot, Gravrusttjern, hardwood, 2.IX.1978 Høgholen 832/78 (O 165333). Østfold: Aremark, Tjøstøl, P. abies, 24.X.2011 Svantesson 1008 (O). Sør-Trøndelag: Tydal, Hilmo, P. abies, 22.IX.2011 Svantesson 368 (O). Nord-Trøndelag: Snåsa, Blåfjella, P. abies, 26.IX.2011 J. Nordén 9601* (O F-248016), Svantesson 608*(O). Russia. Khabarovsk Reg.: Solnechnyi Dist., Igdomi, Picea ajanensis, 3.IX.2016 Spirin 10856, 10916 (H). Krasnoyarsk Reg.: Turukhansk Dist., Mirnoye, Pinus sibirica, 20.VIII.2013 Kotiranta 26387* (H). Leningrad Reg.: Boksitogorsk Dist., Chagoda, P. abies, 9.V.2018 Spirin 11886* (H), Kolp', P. abies, 27.VII.2016 Spirin 10376 (H), Vyalgozero, P. abies, 13.VII.2014 Spirin 6936(H); Kirishi Dist., Oblutskoye, dead Trichaptum abietinum on P. abies, 6.VIII.2019 Spirin 12844 (H); Podporozhie Dist., Vazhinka, P. abies, 21.V.2016 Spirin 10101, 10103, 10106, 10108 (H), 5.VI.2016 Spirin 10182 (H). Nizhny Novgorod Reg.: Arzamas Dist., Pustynsky Nat. Res., P. sylvestris, 12.VIII.2015 Spirin 9188a* (H); Lukoyanov 
Dist., Panzelka, P. sylvestris, 17.VIII.2015 Spirin 9481*(H), Razino, U. glabra, 18.VIII.2015 Spirin 9483* (H); Sharanga Dist., Kilemary Nat. Res., P. abies, 23.VIII.2019 Spirin 12972 (H), 24.VIII.2019 Spirin 13046 (H). Tyumen Reg.: YamaloNenets Autonomous Dist., W of Labytnangi, decorticated coniferous log, 24.VIII.1996 Kotiranta 12721 (H). Zabaikalie: Duldurga Dist., Alkhanai, Pinus sp., 26.VIII.2010 Kotiranta 29616* (H); Kyra Dist., Verkhnii Bukukun, decorticated coniferous log, 9.IX.2010 Kotiranta 29923 (H). Slovenia. Gorenjska: Kranj, Njivica, P. abies, 25.IX.2019 Spirin 13147*(H); Kranjska Gora, Vršič, Larix decidua, 5.VI.2019 Spirin 12548*(H), Zelenci Spring, P. abies, 25.IX.2019 Spirin 13190 (H); Bohinj, Lipanca, L. decidua, 26.IX.2019 Spirin 13269 (H), P. abies, 6.VI.2019 Spirin 12623*(H), 26.IX.2019 Spirin $13244(\mathrm{H}), P$. mugo, 6.VI.2019 Spirin 12650 (H), Mrežce, P. abies, together with Membranomyces delectabilis, 26.IX.2019 Spirin 13204 (H); Mojstrana, Triglavska Bistrica, P. abies, 28.IX.2019 Spirin 13468 (H). Sweden. Västra Götaland: Strömstad, Hästeskede, $P$. abies, 31.X.2011 J. Nordén 10035 (O). Ukraine. Kharkov Reg.: Krasnokutsky Dist., Slobozhansky Nat. Park, Quercus robur, 2.VII.2015 Savchenko 72* (CWU 7558). USA. Washington: Clallam Co., Hurricane Ridge, Abies lasiocarpa, 19.X.2014 Spirin 8740* (H), La Push, Pseudotsuga menziesii, 8.X.2014 Spirin $8262 *(\mathrm{H})$.

Basidiodendron walleynii Spirin, V. Malysheva \& Schoutteten, sp. nov. - Figs. 4, 5 and 7

MB 838731

Holotype. Russia. Nizhny Novgorod Reg.: Lukoyanov Dist., Sanki, 54.841N 44.235E, Quercus robur (rotten stump), 20.VIII.2015 Spirin $9697^{*}$ (H, isotype - LE).

Etymology: After Ruben Walleyn, a Flemish mycologist and collector of the first modern specimen of this species.

Basidiocarps effused, smooth, first waxy, pruinose, whitish or greyish, then continuous, arid, rather soft, cream-coloured to pale ochraceous, opaque, $0.02-0.1 \mathrm{~mm}$ thick, covering a few $\mathrm{cm}$, margin gradually thinning-out. Hyphal structure monomitic, hyphae clamped, easily collapsing, freely spaced; subicular hyphae thin- or slightly thick-walled, subparallel, 2.5-3 $\mu \mathrm{m}$ diam, subhymenial hyphae thin- or slightly thickwalled, ascending, 2.5-3 $\mu \mathrm{m}$ diam. Gloeocystidia abundant, moderately tapering or tubular-clavate, rarely subcapitate, projecting up to $20 \mu \mathrm{m}$, hyaline to very pale yellowish or brownish, (14.5) 15-38 (39) $\times$ (4.6) 5.0-10.7 (11.2) $\mu \mathrm{m}(n$ $=67 / 5)$, often in groups of 3-5. Basidia four-celled, ovoid to broadly suburniform, $(10.0) 10.8-15.7(17.2) \times(7.0) 7.2-11.1$ (11.8) $\mu \mathrm{m}(n=70 / 5)$, sterigmata gradually tapering, up to 10 $\times 2.5 \mu \mathrm{m}$; involucres indistinct. Basidiospores warted, thinwalled, compressed-subglobose or globose, (5.0) 5.1-7.0 $\times$ (5.2) 5.3-7.2 (7.3) $\mu \mathrm{m}(n=120 / 4), \mathrm{L}=5.87-6.17, \mathrm{~W}=$ 6.07-6.38, Q' $=0.9-1.0, \mathrm{Q}=0.96-0.98$, apiculus prominent, regular, sometimes slightly asymmetric, up to $2.5 \times 2 \mu \mathrm{m}$.
Distribution and ecology. Europe (Belgium, France, Russia); decorticated wood of deciduous trees in temperate forests.

Remarks. Basidiodendron walleynii produces rather thick, opaque, soft basidiocarps reminiscent more of Hyphoderma or Peniophorella spp. than other Basidiodendron species. It differs from two other European Basidiodendron species with warted basidiospores, i.e., B. cinerellum and B. trachysporum, in having a non-gelatinized hymenium, a well-differentiated subhymenium consisting of ascending hyphae, and clearly projecting, variably shaped gloeocystidia. Basidiodendron walleynii is a temperate species; it occurs on rather tough, decorticated wood of deciduous trees (Castanea, Erica, Quercus).

Specimens examined (paratypes). Belgium. Vlaams-Brabant: Hoeilaart, Zoniënwoud, Kersselaerspleyn, Q. robur, 24.IX.2002 Walleyn 3081* (GENT). France. Aveyron: Boutaran, dead Inonotus dryadeus, 12.V.1914 Galzin 15320 (herb. Bourdot 13969) (PC 0706667); Forques, Castanea sativa, 20.IV.1912 Galzin 11270 (herb. Bourdot 9068) (PC 0706671), Galzin 11276 (herb. Bourdot 9067) (PC 0706662); Le Rec, C. sativa, XI.1922 Galzin 27569 (herb. Bourdot 35049) (PC 0706663); Loubotis, C. sativa, 13.XI.1913 Galzin 14136 (herb. Bourdot 12434) (PC 0706670); Matavalès, C. sativa, 20.IV.1912 Galzin 11329 (herb. Bourdot 9069) (PC 0706664); Mazet Bas, $C$. sativa, 16.VII.1912 Galzin 11650 (herb. Bourdot 9070) (PC 0084212), 5.IX.1912 Galzin 11881 (herb. Bourdot 9071) (PC 0084223), 23.X.1915 Galzin 18653 (herb. Bourdot 19447) (PC 0706659), XII.1915 Galzin 19135 (herb. Bourdot 15528) (PC 0706661), 5.XI.1916 Galzin 20597 (herb. Bourdot 19365) (PC 0706668), 24.XII.1916 Galzin 21277 (herb. Bourdot 19356) (PC 0706669); Travès, Erica arborea, 12.II.1912 Galzin 10712 (herb. Bourdot 9063) (PC 0084224).

\section{Basidiodendron widdringtoniae Spirin, V. Malysheva \&} Ryvarden, sp. nov. - Figs. 4 and 8

MB 838732

Holotype. Malawi. Southern Prov.: Mulanje, Lichenya Plateau, 15.914S 35.589E, Widdringtonia whytei (rotten decorticated $\log$ ), 9-10.III.1973 Ryvarden 11307a* (O, isotypes - H, LE).

Etymology: After Widdringtonia, the host tree of the species.

Basidiocarps effused, smooth, waxy, pruinose-reticulate, whitish or greyish, $0.01-0.02 \mathrm{~mm}$ thick, covering a few $\mathrm{cm}$, margin gradually thinning-out. Hyphal structure monomitic, hyphae clamped, partly glued together; subicular hyphae thin- to slightly thick-walled, subparallel, 2.5-3 $\mu \mathrm{m}$ diam, subhymenial hyphae thin- to slightly thick-walled, ascending or interwoven, 2-3 $\mu \mathrm{m}$ diam. Gloeocystidia abundant, clearly tapering, slightly projecting, hyaline or yellowish to brownish, (12) 13.5-21 (24) $\times$ (3.4) 3.9-5.9 (6.0) $\mu \mathrm{m}$ $(n=14 / 1)$. Basidia two - four-celled, ovoid to broadly 
suburniform, (10.2) 11.7-14.2 (14.8) × (8.1) 8.2-10.8 (12.0) $\mu \mathrm{m}(n=20 / 1)$, sterigmata gradually tapering, up to $8 \times$ 2-2.5 $\mu \mathrm{m}$; involucres well-developed, often totally covering basidial cells (except sterigmata); basidia in older hymenium gluedin groups of 3-4 and occasionally embedded in gelatinous matrix. Basidiospores spiny (spines up to $0.3 \mu \mathrm{m}$ long), thin-walled, compressed-subglobose or globose, (5.8) 5.9-6.8 × (6.0) 6.1-7.1 (7.2) $\mu \mathrm{m}(\mathrm{n}=30 / 1), \mathrm{L}=6.24, \mathrm{~W}=$ $6.48, Q^{\prime}=0.9-1.0, \mathrm{Q}=0.96$, apiculus prominent, regular, sometimes asymmetric, up to $1.5 \times 1.5 \mu \mathrm{m}$.

Distribution and ecology. So far known from the type locality; rotten wood of conifers (Widdringtonia whytei).

Remarks. Basidiodendron widdringtoniae is morphologically most similar to $B$. mexicanum occurring on coniferous wood in Mexico. It differs from the latter species mainly due to thinner basidiocarps and shorter gloeocystidia. However, our material is too scanty to assure these differences are not age-dependent. Therefore, the two species must for the time being be separated by different DNA sequences and distribution areas.

\section{Discussion}

In this study, we present the taxonomy of twelve species of the B. caesiocinereum complex of which eight are described as new to science. Our study focused mainly on temperateboreal Europe, while sampling from North America and East Asia was occasional. More data may reveal other representatives of the B. caesiocinereum group in those areas, as well as in warm-temperate to Mediterranean regions of Europe. A few available environmental sequences support this suggestion. In fact, the species diversity in this complex could be even higher because $B$. caesiocinereum and $B$. spinosum s. lato were reported from Macaronesia (Roberts and Spooner 2004), North and Central Africa (Malençon 1954; Roberts 2001), the Caribbean (Roberts 2006, 2008), Central and South America (Kisimova-Horovitz et al. 1997; Roberts 2003), and New Zealand (McNabb 1969). This material awaits proper taxonomic revisions.

Despite rather uniform anatomical traits, members of the B. caesiocinereum complex are morphologically distinguishable, although microscopy requires the use of phase contrast illumination and Cotton Blue as mounting medium. Only this combination allows the verification of outgrowths on the basidiospore wall and an identification of their form (warts or spines). As stated above, the basidiospore ornamentation is a key morphological character in this group. Combined with other morphological features (basidiocarp colour and consistency, size of basidia and gloeocystidia, arrangement of hymenial cells), as well as ecological and geographic data, it facilitates the species recognition.
Key for species currently recognized in the Basidiodendron caesiocinereum complex

1. Basidiospores smooth............................................................

$1^{*}$. Basidiospores ornamented ................................................

2. Basidiospores 5.1-7.8 $\times 5.3-8.1 \mu \mathrm{m}$, basidia $12-24 \times 7-11.5 \mu \mathrm{m}$, occasionally with a distinct stalk-like base. Eurasia, almost exclusively on ang iosperms. B. caesiocinereum $2^{*}$. Basidiospores on average smaller, 5.1-6.8 $\times 5.2-7 \mu \mathrm{m}$, basidia $10-18 \times 7-10.5 \mu \mathrm{m}$, devoid of stalk-like base .............. 3. Eurasia, almost exclusively on gymnosperms (Abies, Pic ea). B. glaucum

3*. North America, exclusively on angiosperms. B. robenae

4. Basidiospores warted.......................................................... 5

4*. Basidiospores spiny ......................................................

5. Basidia glued in groups. Europe, mostly on gymnosperms B. cinerellum

$5^{*}$. Basidia exposed. On various hosts.

. .6

6. Basidiocarps arid, opaque. Subhymenial hyphae wellvisible, ascending. Gloeocystidia tapering or tubular-clavate, $15-38 \times 5-11 \mu \mathrm{m}$. Temperate Europe, exclusively on angiosperms (Erica, Castanea, Quercus) B. walleynii $6^{*}$. Basidiocarps waxy, hymenium often gelatinized. Subhymenium hyphae usually poorly discernible, glued together. Gloeocystidia tapering, 13-45 ×3-8 $\mu \mathrm{m}$. Eurasia and North America, mostly on gymnosperms 7

7. Basidiospores distinctly warted, 4.8-7.4 $\times 5.0-7.8 \mu \mathrm{m}$. Eurasia and North America.. B. trachysporum

7*. Basidiospores minutely warted, 5.0-6.2 $\times 5.2-6.5 \mu \mathrm{m}$. North American North-West B. inconspicuum 8. Basidia glued in groups. South Africa (Malawi), on gymnosperms... B. widdringtoniae

8*. Basidia exposed. On various hosts....................................

9. Basidiospores with spines up to $0.6 \mu \mathrm{m}$ long....................10

$9 *$. Basidiospores with spines up to $0.4 \mu \mathrm{m}$ long ................11

10. Basidia 9-10 × 6-7.5 $\mu \mathrm{m}$, basidiospores 4.6-5.2 $\times 4.8-$ $5.8 \mu \mathrm{m}$. Oceania B. spinosum

10*. Basidia 13-18 × 9-12 $\mu \mathrm{m}$, basidiospores $6.0-7.9 \times$ 6.2-8.2 $\mu \mathrm{m}$. Europe ……………………….... B. groningae

11. Basidia $12-15.5 \times 9.5-12 \mu \mathrm{m}$, basidiospores 5.9-7.3 × 6.1-7.4 $\mu \mathrm{m}$. Mexico (highlands), on gymnosperms B. mexicanum

$11^{*}$. Basidia 15-24× 10-12 $\mu \mathrm{m}$, basidiospores 6.9-8.2 × 7.1-8.9 $\mu \mathrm{m}$. Mexico (subtropical zone), on ferns (Cyathea).

B. spiculosum

Additional specimens examined

Basidiodendron eyrei. Netherlands. Gelderland: Hoge Veluwe, decayed wood, 16.XI.2019 Schoutteten 19-411* (GENT); Groningen: Nieuweschans, Houwingaham, 
decayed wood, 24.X.2018 Enzlin 18-101* (GENT); Oude Pekela, Emergobos, decayed wood, 7.X.2018 Enzlin 18-103* (GENT); Ter Apel, Roelage Bos, P. abies, 22.X.2013 Enzlin 13-100* (GENT).

Acknowledgements We are very indebted to the curators of herbaria FH, PC, NY, TU, TAAM and CWU, who sent us valuable specimens for loan. Myriam de Haan (Meise Botanical Garden, Ghent, Belgium) kindly provided us with SEM photographs of B. groningae.

Author contribution All authors contributed to the study conception and design. Material preparation, data collection and analysis were initially performed by Viacheslav Spirin, Vera Malysheva, Otto Miettinen, Ilya Viner and Karl-Henrik Larsson. The authors Nathan Schoutteten, Jenni Nordén, Heikki Kotiranta, Leif Ryvarden, Annemieke Verbeken and Michael Weiß provided further important material and/or sequences. The first draft of the manuscript was written by Viacheslav Spirin and all authors commented on previous versions of the manuscript. All authors read and approved the final manuscript.

Funding Open access funding provided by University of Helsinki including Helsinki University Central Hospital. The research was supported by Norwegian Biodiversity Information Centre (projects "A survey of Norwegian jelly fungi", grant number knr. 44-15, the authors KHL and VS, "Wood-inhabiting corticioid homo- and hetero-basidiomycetes in Norway", grant number knr. 59-12, and "International workshop on corticioid heterobasidiomycetes in Northern Europe", grant number knr. 28-13, the authors JN, KHL, VS), Academy of Finland research grant (number 315927, the authors VS and OM) Komarov Botanical Institute, Russian Academy of Sciences (project AAAAA19-119020890079-6, the author VM), and Flemish Research Council (FWO) (Fundamental Research Fellowship, grant number 11E0420N, the author NS).

Data availability DNA sequences used in the present study are available in GenBank. Alignments were deposited in TreeBase. Fungal specimens are stored in public herbaria (as indicated under Specimens examined)

\section{Declarations}

Ethics approval Not applicable

\section{Conflict of interests None.}

Open Access This article is licensed under a Creative Commons Attribution 4.0 International License, which permits use, sharing, adaptation, distribution and reproduction in any medium or format, as long as you give appropriate credit to the original author(s) and the source, provide a link to the Creative Commons licence, and indicate if changes were made. The images or other third party material in this article are included in the article's Creative Commons licence, unless indicated otherwise in a credit line to the material. If material is not included in the article's Creative Commons licence and your intended use is not permitted by statutory regulation or exceeds the permitted use, you will need to obtain permission directly from the copyright holder. To view a copy of this licence, visit http://creativecommons.org/licenses/by/4.0/.

\section{References}

Bourdot H, Galzin A (1927) Hyménomycètes de France. Hetérobasidiés - Homobasidiés gymnocarpes. Sceaux

Bourdot H, Maire L (1920) Notes critiques sur quelques Hyménomycètes nouveaux ou peu connus. Bull Soc Mycol France 36:69-85

Gardes M, Bruns TD (1993) ITS primers with enhanced specificity for basidiomycetes - applications to the identification of mycorrhizae and rusts. Mol Ecol 2:113-118

Gilbertson RL (1974) Fungi that decay ponderosa pine. University of Arizona Press, Tucson

Höhnel F, Litschauer V (1908) Beiträge zur Kenntnis der Corticieen. III. Sitzungsb. Kais. Akad. Wissenschaften Wien, Mat - Nat Klasse Abt. I, 117:1081-1124

Kisimova-Horovitz L, Oberwinkler F, Gómez LD (1997) Basidiomicetos resupinados de Costa Rica. Exidiaceae (Tremellales). Rev Biol Trop 45:1325-1347

Kozlov AM, Darriba D, Flouri T, Morel B, Stamatakis A (2019) RAxML-NG: A fast, scalable, and user-friendly tool for maximum likelihood phylogenetic inference. Bioinformatics btz305. https:// doi.org/10.1093/bioinformatics/btz305

Kumar S, Stecher G, Li M, Knyaz C, Tamura K (2018) MEGA X: Molecular Evolutionary Genetics Analysis across computing platforms. Mol Biol Evol 35:1547-1549

Landvik S (1996) Neolecta, a fruit-body producing genus of the basal ascomycetes, as shown by SSU and LSU rDNA sequences. Mycol Res 100:199-202

Luck-Allen R (1963) The genus Basidiodendron. Can J Bot 41:1025-1052

Malençon G (1954) Prodrome d'une Flore Mycologique du Moyen Atlas. 2e Contribution. Bull Soc Mycol France 70:117-156

McNabb RFR (1969) New Zealand Tremellales - III. N Z J Bot 7:241-261

Oberwinkler F (1963) Niedere Basidiomyceten aus Südbayern. III. Die Gattung Sebacina Tul. s.1. Ber Bayerischen Bot Ges 36:41-55

Rambaut A, Suchard MA, Xie D, Drummond AJ (2014) Tracer v1.6. http://tree.bio.ed.ac.uk/software/tracer/ Accessed 20 Dec 2020

Rehner SA, Buckley E (2005) A Beauveria phylogeny inferred from nuclear ITS and EF1-alpha sequences: evidence for cryptic diversification and links to Cordyceps teleomorphs. Mycologia 97:84-98

Roberts P (2001) Heterobasidiomycetes from Korup National Park, Cameroon. Kew Bull 56:163-187

Roberts P (2003) Heterobasidiomycetes from Rancho Grande, Venezuela. Mycotaxon 87:25-41

Roberts P (2006) Caribbean heterobasidiomycetes: 2 Jamaica. Mycotaxon 96:83-107

Roberts P (2008) Caribbean heterobasidiomycetes: 3 British Virgin Islands. Mycotaxon 105:137-147

Roberts P, Spooner B (2004) Heterobasidiomycetes from the Azores. Kew Bull 59:95-101

Ronquist F, Teslenko M, van der Mark P, Ayres DL, Darling A, Höhna S, Larget B, Liu L, Suchard MA, Huelsenbeck JP (2012) MrBayes 3.2: efficient Bayesian phylogenetic inference and model choice across a large model space. Syst Biol 61:539-542

Stein F (1969) Particle size measurements with phase contrast microscopy. Powder Technol 2:327-334

Spirin V, Malysheva V, Mendes-Alvarenga RL, Kotiranta H, Larsson $\mathrm{KH}$ (2020) Studies in Basidiodendron eyrei and similar-looking taxa (Auriculariales, Basidiomycota). Botany 98:623-638

Thiers B (2020) Index Herbariorum: a global directory of public herbaria and associated stuff [continuosly updated]. New York Botanical Garden's Virtual Herbarium. http://sweetgum.nybg.org/ ih. Accessed 20 Dec 2020 
Vilgalys R, Hester M (1990) Rapid genetic identification and mapping of enzymatically amplified ribosomal DNA from several Cryptococcus species. J Bacteriol 172:4238-4246

Weiß M, Oberwinkler F (2001) Phylogenetic relationships in Auriculariales and related groups - hypotheses derived from nuclear ribosomal DNA sequences. Mycol Res 105:403-415

Wells K (1959) Studies of some Tremellaceae III. The genus Bourdotia. Mycologia 51:540-563

Wells K, Raitviir A (1975) The species of Bourdotia and Basidiodendron (Tremellaceae) of the USSR. Mycologia 67:904-922
White TJ, Bruns T, Lee S, Taylor J (1990) Amplification and sequencing of fungal ribosomal RNA genes for phylogenetics. In: Innis MA et al (eds) PCR protocols A guide to methods and applications. Academic Press, San Diego, pp 315-322

Wojewoda W (1981) Mała flora grzybów. Tom 2. Krakow, Państwowe Wydawnictwo Naukowe Warsaw

Publisher's note Springer Nature remains neutral with regard to jurisdictional claims in published maps and institutional affiliations. 